Homology, Homotopy and Applications, vol.5(1), 2003, pp.549-599

\title{
A MODEL CATEGORY FOR THE HOMOTOPY THEORY OF CONCURRENCY
}

\author{
PHILIPPE GAUCHER
}

(communicated by Mark Hovey)

\begin{abstract}
We construct a cofibrantly generated model structure on the category of flows such that any flow is fibrant and such that two cofibrant flows are homotopy equivalent for this model structure if and only if they are S-homotopy equivalent. This result provides an interpretation of the notion of S-homotopy equivalence in the framework of model categories.
\end{abstract}

\section{Geometric models of concurrency}

Algebraic topological models have been used now for some years in concurrency theory (concurrent database systems and fault-tolerant distributed systems as well) [23]. The earlier models, progress graph (see [6] for instance) have actually appeared in operating systems theory, in particular for describing the problem of "deadly embrace" (as E. W. Dijkstra originally put it in [8], now more usually called deadlock) in "multiprogramming systems". They are used by J. Gunawardena in [25] as an example of the use of homotopy theory in concurrency theory. Later V. Pratt introduced another geometric approach using strict globular $\omega$-categories in [32]. Some of his ideas would be developed in an homological manner in E. Goubault's PhD $[\mathbf{2 2}]$, using bicomplexes of modules. The $\omega$-categorical point of view would be developed by the author mainly in $[\mathbf{1 3}][\mathbf{1 4}][\mathbf{1 5}][\mathbf{1 6}]$ using the equivalence of categories between the category of strict globular $\omega$-categories and that of strict cubical $\omega$ categories [1]. The mathematical works of R. Brown et al. [5] [4] and of R. Street [34] play an important role in this approach.

The $\omega$-categorical approach also allowed to understand how to deform higher dimensional automata (HDA) modeled by $\omega$-categories without changing their computer-scientific properties (deadlocks, unreachable states, schedules of execution, final and initial points, serializability). The notions of spatial deformation and of temporal deformation of HDA are indeed introduced in [12] in an informal way.

Another algebraic topological approach of concurrency is that of local po-space introduced by L. Fajstrup, E. Goubault and M. Raussen. A local po-space is a gluing of topological spaces which are equipped with a closed partial ordering representing the time flow. They are used as a formalization of higher dimensional automata

Received August 9, 2003, revised November 30, 2003; published on December 26, 2003. 2000 Mathematics Subject Classification: 55P99, 68Q85.

Key words and phrases: concurrency, higher dimensional automaton, homotopy, closed monoidal structure, cofibration, compactly generated topological space, cofibrantly generated model category (C) 2003, Philippe Gaucher. Permission to copy for private use granted. 
which model concurrent systems in computer science. Some algorithms of deadlock detection in PV diagrams have been studied within this framework [10].

The notion behind all these geometric approaches is the one of precubical set. Roughly speaking, a $n$-dimensional cube $[0,1]^{n}$ represents the concurrent execution of $n$ independant processes. A precubical set is a family of sets $\left(K_{n}\right)_{n \geqslant 0}$ (the elements of $K_{n}$ being called the $n$-dimensional cubes) together with face operators

$\partial_{i}^{\alpha}: K_{n+1} \longrightarrow K_{n}$ for $1 \leqslant i \leqslant n$ and with $\alpha \in\{-,+\}$ satisfying $\partial_{i}^{\alpha} \partial_{j}^{\beta}=\partial_{j-1}^{\beta} \partial_{i}^{\alpha}$ for $i<j$. These face operators encode how the $n$-cubes are located with respect to one another in the precubical set. The prefix "pre" means that there are no degeneracy maps at all in the data. R. Cridlig presents in [7] an implementation with CaML of the semantics of a real concurrent language in terms of precubical sets, demonstrating the relevance of this approach. Since this category is sufficient to model HDA, why not deal directly with precubical sets ? Because the category of precubical sets is too poorly structured. For instance there are not enough morphisms to model temporal deformations (see also the introduction of [14] for some further closely related reasons).

In [20], some particular cases of local po-spaces are introduced by E. Goubault and the author: the globular $C W$-complexes. The corresponding category is big enough to model all HDA. Moreover the notion of spatial and temporal deformations can be modeled within this category. It became possible to give a precise mathematical definition of two globular CW-complexes to be S-homotopy equivalent and T-homotopy equivalent (S for space and $\mathrm{T}$ for time !). By localizing with respect to the S-homotopy and T-homotopy equivalences, one obtains a new category, that of dihomotopy types, whose isomorphism classes are globular $\mathrm{CW}$-complexes having the same computer scientific properties. It then became possible to study concurrency using only this quotient category of dihomotopy types.

Not only globular complexes allow to model dihomotopy, but they also allow to take out pathological situations appearing in the local po-space framework and which are meaningless from a computer scientific viewpoint. For example, the rational numbers $\mathbb{Q}$ equipped with the usual ordering is a local po-space and the total disconnectedness of $\mathbb{Q}$ means nothing in this geometric approach of concurrency.

The purpose of this paper is the introduction of a new category, the category of flows, in which it will be possible to embed the category of globular CW-complexes and in which it will be possible to define both the class of S-homotopy and Thomotopy equivalences. Due to the length of this work, the construction and the study of the functor from the category of globular CW-complexes to that of flows is postponed to another paper.

Figure 1 is a recapitulation of the geometric models of concurrency, including the one presented in this paper.

\section{Outline of the paper}

Section 4 defines the category of flows Flow after a short introduction about compactly generated topological spaces. It is proved that Flow is complete and cocomplete. Several particular and important examples of flows are also introduced. 


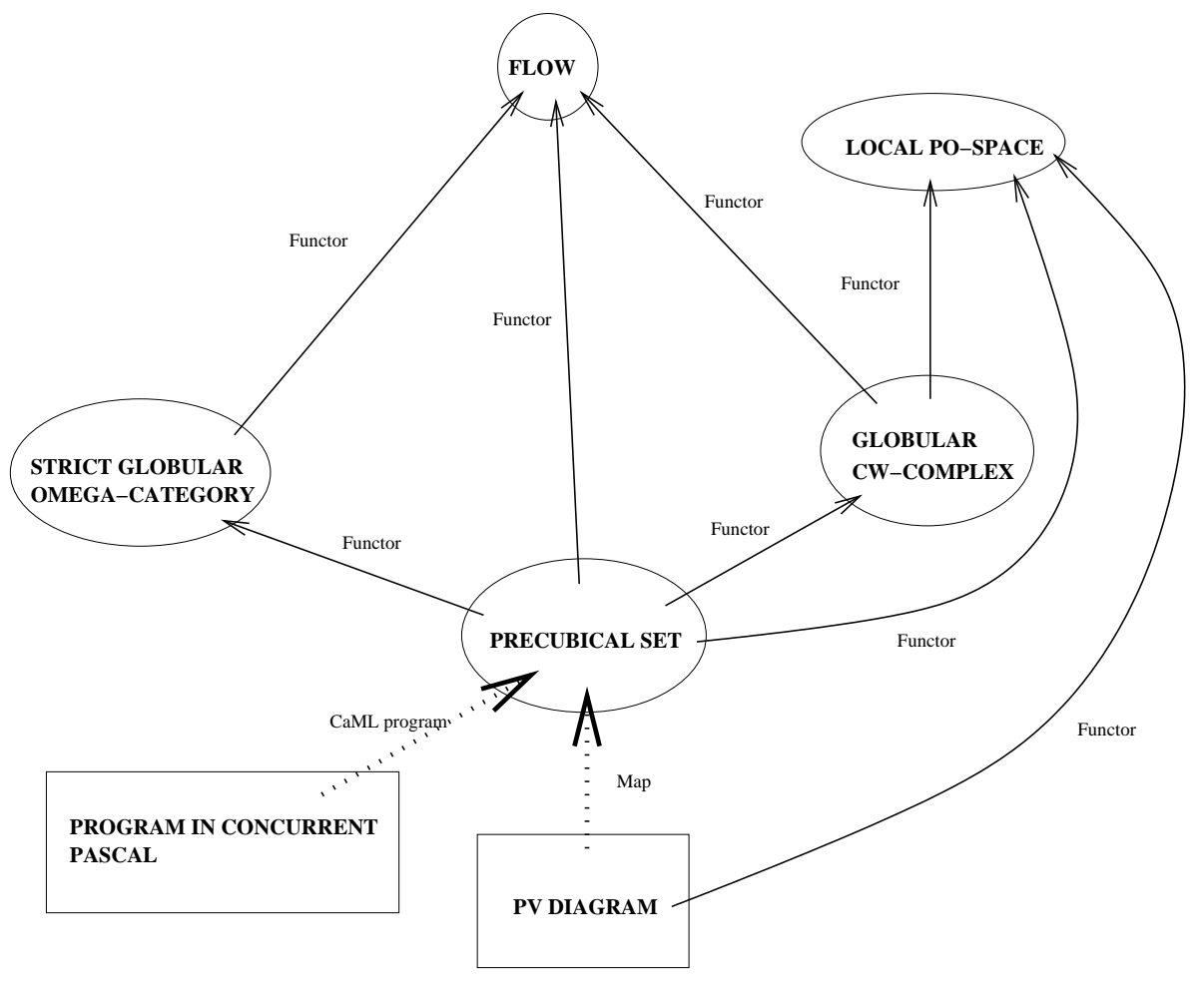

Figure 1: Comparison of geometric models of HDA

Section 5 is devoted to proving that for any flow $Y$, the functor $\mathbf{F L O W}(-, Y)$ from the opposite of the category of flows to that of topological spaces commutes with all limits where $\mathbf{F L O W}(X, Y)$ is the set of morphisms of flows from $X$ to $Y$ endowed with the Kelleyfication of the compact-open topology. This fact will be of crucial importance in several places of the paper. This result turns out to be difficult to establish since the underlying topological space of a colimit of flows is in general not isomorphic to the colimit of the underlying topological spaces. This result actually requires the introduction of the category of non-contracting topological 1-categories and of a closed monoidal structure on it. Section 6 shows that any flow is a canonical colimits of globes and points. This is a technical lemma which is also of importance for several proofs of this paper. Section 7 defines the class of S-homotopy equivalences in the category of flows. The associated cylinder functor is constructed. Section 8 is devoted to an explicit description of $U \otimes X$ for a given topological space $U$ and a given flow $X$. Section 9 describes a class of morphisms of flows (the ones satisfying the S-homotopy extension property) which are closed by pushouts and which contains useful examples as the inclusion $\operatorname{Glob}(\partial Z) \longrightarrow \operatorname{Glob}(Z)$ where $(Z, \partial Z)$ is a NDR pair of topological spaces. The main result of Section 10 is that any morphism of flows satisfying the S-homotopy 
extension property induces a closed inclusion of topological spaces between the path spaces. This allows us to prove in Section 11 that the domains of the generating cofibrations and of the generating trivial cofibrations of the model structure are small relatively to the future class of cofibrations of the model structure. Section 11 is therefore the beginning of the construction of the model structure. Section 12 recalls some well-known facts about cofibrantly generated model categories. Section 13 characterizes the fibrations of this model structure. Section 14 explains why it is necessary to add to the set of generating cofibrations the morphisms of flows $C$ : $\varnothing \longrightarrow\{0\}$ and $R:\{0,1\} \longrightarrow\{0\}$. Section 15 provides an explicit calculation of the pushout of a morphism of flows of the form $\operatorname{Glob}(\partial Z) \longrightarrow \operatorname{Glob}(Z)$. This will be used in Section 16. The main result of Section 15 is that if $\partial Z \longrightarrow Z$ is an inclusion of a deformation retract, then any morphism of flows which is a pushout of $\operatorname{Glob}(\partial Z) \longrightarrow \operatorname{Glob}(Z)$ induces a weak homotopy equivalence between path spaces. Section 16 and Section 17 conclude the construction of the model structure recapitulated in Section 18. Section 19 checks that two cofibrant-fibrant flows are homotopy equivalent for this model structure if and only if they are Shomotopy equivalent.

\section{Warning}

This paper is the first part of a work which aims at introducing a convenient categorical setting for the homotopy theory of concurrency. This part is focused on the category of flows itself, its basic properties, the notion of S-homotopy equivalence, weak or not, and the model structure. The relation between the category of globular CW-complexes and the one of flows is explored in [17]. A detailed abstract (in French) of this work can be found in [18] and [19].

\section{The category of flows}

\subsection{Preliminaries about the compactly generated topological spaces}

This section is a survey about compactly generated spaces which gives enough references for the reader not familiar with this subject. Cf. $[\mathbf{3}],[\mathbf{3 0}]$ and the appendix of $[\mathbf{2 8}]$.

By a compact space, we mean a compact Hausdorff topological space. Let $\mathcal{T}$ be the category of general topological spaces with the continuous maps as morphisms.

Definition 4.1. A continuous map $f: A \longrightarrow B$ is an inclusion of spaces if $f$ is one-to-one and if the canonical set map

$$
\operatorname{Top}(Z, A) \longrightarrow\{g \in \operatorname{Top}(Z, B), g(Z) \subset f(A)\}
$$

induced by the mapping $g \mapsto f \circ g$ is a bijection of sets. In other terms, a continuous map $f: A \longrightarrow B$ is an inclusion of spaces if for any set map $g: Z \longrightarrow A$ such that $f \circ g$ is continuous, then $g$ is continuous.

Definition 4.2. A continuous map $f: A \longrightarrow B$ is closed if for any closed subset $F$ of $A$, the subset $f(F)$ is closed in $B$. 
Definition 4.3. A quotient map is a continuous map $f: X \longrightarrow Y$ which is onto and such that $U \subset Y$ is open if and only if $f^{-1}(U)$ is open in $X$. In other term, $Y$ is given with the final topology associated to $f$.

Definition 4.4. A $k$-space $X$ is a topological space such that for any continuous map $f: K \longrightarrow X$ with $K$ compact, $U \subset X$ is open (resp. closed) if and only if $f^{-1}(U)$ is open (resp. closed) in $K$. The corresponding category with the continuous maps as morphisms is denoted by $k$ Top.

A topological space $X$ is a $k$-space if and only if there exists a disjoint sum of compacts $\bigoplus_{i \in I} K_{i}$ and a quotient map $\bigoplus_{i \in I} K_{i} \longrightarrow X[\mathbf{3}]$. The inclusion functor $k$ Top $\longrightarrow \mathcal{T}$ has a right adjoint and a left inverse $k: \mathcal{T} \longrightarrow k$ Top which is called the Kelleyfication functor. The category $k$ Top is complete and cocomplete where colimits are taken in $\mathcal{T}$ and limits are taken by applying $k$ to the limit in $\mathcal{T}[\mathbf{3 3}]$ [29]. The identity map $k(X) \longrightarrow X$ is continuous because the topology of $k(X)$ contains more opens than the topology of $X$.

Definition 4.5. A topological space $X$ is weak Hausdorff if and only if for any continuous map $f: K \longrightarrow X$ with $K$ compact, the subspace $f(K)$ is closed in $X$.

If $X$ is a $k$-space, then $X$ is weak Hausdorff if and only if its diagonal $\Delta X=$ $\{(x, x) \in X \times X\}$ is a closed subspace of $X \times X$, the latter product being taken in $k$ Top [31]. If $X$ is a weak Hausdorff topological space, then $k(X)$ is still weak Hausdorff.

If $X$ is a weak Hausdorff topological space, then $X$ is a $k$-space if and only if $X \cong \lim _{K \subset X} K$ as topological space where $K$ runs over the set of compact subspaces of $X$ : a subset $F$ of $k(X)$ is closed (resp. open) if and only if for any compact $C$ of $X, F \cap C$ is a closed (resp. open) subspace of $X$.

Definition 4.6. A compactly generated topological space is by definition a weak Hausdorff $k$-space. The corresponding category with the continuous maps as morphims is denoted by Top.

Let $w H$ be the category of weak Hausdorff topological spaces. Generally colimits in $w H$ do not coincide with colimits in $\mathcal{T}$. But

Proposition 4.7. [28] A transfinite composition of injections and pushouts of closed inclusions of compactly generated topological spaces is still weak Hausdorff (and therefore a compactly generated topological space).

Proposition 4.8. [31] [29] The inclusion functor $w H \longrightarrow \mathcal{T}$ has a left adjoint $H$. If $X$ is a $k$-space and if $\mathcal{R}$ is an equivalence relation, then $H(X / \mathcal{R})$ is equal to $X / \overline{\mathcal{R}}$ where the topological closure $\overline{\mathcal{R}}$ of $\mathcal{R}$ is defined as the intersection of all equivalence relations containing $\mathcal{R}$ and whose graph is closed in $X \times X$. In particular, if the graph of $\mathcal{R}$ is closed in $X \times X$, then $X / \mathcal{R}$ is weak Hausdorff.

Proposition 4.9. [33] [29] If $i \mapsto X_{i}$ is any small diagram in Top, then the limit in Top coincides with the Kelleyfication of the limit in $\mathcal{T}$ and with the Kelleyfication of the limit in $w H$. Moreover the underlying set of this limit coincides with the limit in the category of sets of the underlying sets of the $X_{i}$. 
If $X$ is a weak Hausdorff topological space, then a subset $Y$ of $X$ equipped with the relative topology is weak Hausdorff as well. If $X$ is a compactly generated topological space, then a subset $Y$ of $X$ equipped with the relative topology is then weak Hausdorff. But it is not necessarily a $k$-space. To get back a $k$-space, it is necessary to consider the Kelleyfication $k\left(Y_{r}\right)$ of $Y_{r}$ ( $Y$ equipped with the relative topology).

Proposition 4.10. [33] [29] Let us denote by $\mathbf{T O P}(X,-)$ the right adjoint of the functor $-\times X:$ Top $\longrightarrow$ Top. Then

1. If $\operatorname{Cop}(X, Y)$ is the set $\operatorname{Top}(X, Y)$ equipped with the compact-open topology (i.e. a basis of opens is given by the sets

$$
N(C, U):=\{f \in \operatorname{Top}(X, Y), f(C) \subset U\}
$$

where $C$ is any compact subset of $X$ and $U$ any open subset of $Y)$, then there is a natural bijection $\mathbf{T O P}(X, Y) \cong k(C o p(X, Y))$.

2. There is a natural isomorphism of topological spaces

$$
\operatorname{TOP}(X \times Y, Z) \cong \operatorname{TOP}(X, \mathbf{T O P}(Y, Z)) .
$$

3. There are natural isomorphisms of topological spaces

$$
\operatorname{TOP}\left(\underset{i}{\lim } X_{i}, Y\right) \cong \varliminf_{i}^{\lim } \mathbf{T O P}\left(X_{i}, Y\right)
$$

and

$$
\operatorname{TOP}\left(X,{\underset{i}{i}}_{\lim _{i}} Y_{i}\right) \cong{\underset{i}{i}}_{\lim _{i}} \mathbf{T O P}\left(X, Y_{i}\right) .
$$

Similar results can be found in [36] [37] with slightly bigger categories of topological spaces than the one we are using in this paper.

In the sequel, all topological spaces will be supposed to be compactly generated (so in particular weak Hausdorff). In particular all binary products will be considered within this category.

\subsection{Definition of a flow}

Definition 4.11. A flow $X$ consists of a topological space $\mathbb{P} X$, a discrete space $X^{0}$, two continuous maps $s$ and $t$ from $\mathbb{P} X$ to $X^{0}$ and a continuous and associative map

$$
*:\{(x, y) \in \mathbb{P} X \times \mathbb{P} X ; t(x)=s(y)\} \longrightarrow \mathbb{P} X
$$

such that $s(x * y)=s(x)$ and $t(x * y)=t(y)$. A morphism of flows $f: X \longrightarrow Y$ consists of a set map $f^{0}: X^{0} \longrightarrow Y^{0}$ together with a continuous map $\mathbb{P} f: \mathbb{P} X \longrightarrow$ $\mathbb{P Y}$ such that $f(s(x))=s(f(x)), f(t(x))=t(f(x))$ and $f(x * y)=f(x) * f(y)$. The corresponding category will be denoted by Flow.

The continuous map $s: \mathbb{P} X \longrightarrow X^{0}$ is called the source map. The continuous map $t: \mathbb{P} X \longrightarrow X^{0}$ is called the target map. One can canonically extend these two maps to the whole underlying topological space $X^{0} \sqcup \mathbb{P} X$ of $X$ by setting $s(x)=x$ and $t(x)=x$ for $x \in X^{0}$. 


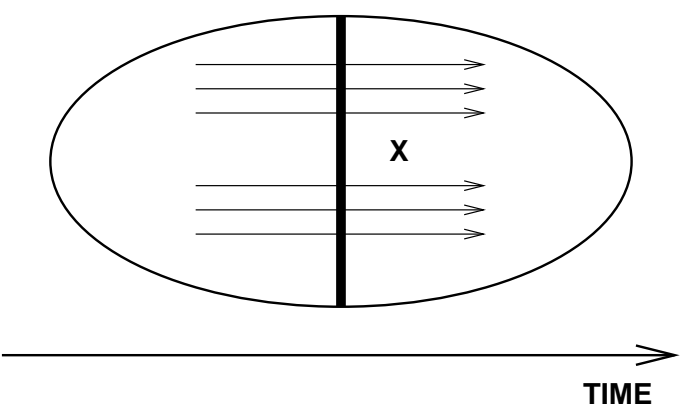

Figure 2: Symbolic representation of $\operatorname{Glob}(X)$ for some topological space $X$

The discrete topological space $X^{0}$ is called the 0 -skeleton of $X$. The 0 -dimensional elements of $X$ are also called states or constant execution paths.

The elements of $\mathbb{P} X$ are called non constant execution paths. If $\gamma_{1}$ and $\gamma_{2}$ are two non-constant execution paths, then $\gamma_{1} * \gamma_{2}$ is called the concatenation or the composition of $\gamma_{1}$ and $\gamma_{2}$. For $\gamma \in \mathbb{P} X, s(\gamma)$ is called the beginning of $\gamma$ and $t(\gamma)$ the ending of $\gamma$.

Notation 4.12. For $\alpha, \beta \in X^{0}$, let $\mathbb{P}_{\alpha, \beta} X$ be the subspace of $\mathbb{P} X$ equipped the Kelleyfication of the relative topology consisting of the non-execution paths of $X$ with beginning $\alpha$ and with ending $\beta$.

Definition 4.13. Let $X$ be a flow. A point $\alpha$ of $X^{0}$ such that there is not any non-constant execution path $\gamma$ with $t(\gamma)=\alpha$ (resp. $s(\gamma)=\alpha$ ) is called an initial state (resp. a final state).

\subsection{The globe of a topological space}

As in [20], but here for the framework of flows, we are going to introduce the notion of globe of a topological space. It will be important both for computer scientific and purely mathematical reasons.

For $X$ a topological space, let Glob $(X)$ be the flow defined by

$$
\text { Glob }(X)^{0}=\{0,1\} \text { and } \mathbb{P G l o b}(X)=X
$$

with $s=0$ and $t=1$ (cf. Figure 2). The Glob mapping induces a canonical functor from the category Top of topological spaces to the category Flow of flows.

As a particular case of globe is that of a singleton. One obtains the directed segment $\vec{I}$. It is defined as follows: $\vec{I}^{0}=\{0,1\}, \mathbb{P} \vec{I}=\{[0,1]\}, s([0,1])=0$ and $t([0,1])=1$.

If $Z_{1}, \ldots, Z_{p}$ are $p$ topological spaces with $p \geqslant 2$, the flow

$$
\operatorname{Glob}\left(Z_{1}\right) * \operatorname{Glob}\left(Z_{2}\right) * \cdots * \operatorname{Glob}\left(Z_{p}\right)
$$

is the flow obtained by identifying the final state of $\operatorname{Glob}\left(Z_{i}\right)$ with the initial state of $\operatorname{Glob}\left(Z_{i+1}\right)$ for $1 \leqslant i \leqslant p-1$. 
Notation 4.14. If $X$ and $Y$ are two flows, let us denote by $\mathbf{F L O W}(X, Y)$ the space of morphisms of flows $\operatorname{Flow}(X, Y)$ equipped with the Kelleyfication of the compact-open topology.

Proposition 4.15. Let $X$ be a flow. Then there is a natural homeomorphism $\mathbb{P} X \cong$ FLOW $(\vec{I}, X)$.

Proof. If we have an element $u$ of $\mathbb{P} X$, consider the morphism of flows $F_{\gamma}$ defined by $F_{\gamma}(0)=s(u), F_{\gamma}(1)=t(u)$ and $F_{\gamma}([0,1])=u$. And reciprocally a morphism $F \in$ Flow $(\vec{I}, X)$ can be mapped on an element of $\mathbb{P} X$ by $F \mapsto F([0,1])$. Hence the bijection between the underlying sets. This bijection is an homemorphism since for any topological space $Z$, one has the homeomorphism $\mathbf{T O P}(\{0\}, Z) \cong Z$.

\subsection{Higher dimensional automaton and flow}

This example is borrowed from [20]. An example of progress graph, that is of higher dimensional automaton, is modeled here as a flow.

The basic idea is to give a description of what can happen when several processes are modifying shared resources. Given a shared resource $a$, we see it as its associated semaphore that rules its behaviour with respect to processes. For instance, if $a$ is an ordinary shared variable, it is customary to use its semaphore to ensure that only one process at a time can write on it (this is mutual exclusion). A semaphore is nothing but a register which counts the number of times a shared object can still be accessed by processes. In the case of usual shared variables, this register is initialized with value 1 , processes trying to access (read or write) on the corresponding variable compete in order to get it first, then the semaphore value is decreased: we say that the semaphore has been locked ${ }^{1}$ by the process. When it is equal to zero, all processes trying to access this semaphore are blocked, waiting for the process which holds the lock to relinquish it, typically when it has finished reading or writing on the corresponding variable: the value of the semaphore is then increased.

When the semaphores are initialized with value one, meaning that they are associated with shared variables accessed in a mutually exclusive manner, they are called binary semaphores. When a shared data (identified with its semaphore) can be accessed by one or more processes, meaning that the corresponding semaphore has been initialized with a value greater than one, it is called a counting semaphore.

Given $n$ deterministic sequential processes $Q_{1}, \ldots, Q_{n}$, abstracted as a sequence of locks and unlocks on (semaphores associated with) shared objects,

$$
Q_{i}=R^{1} a_{i}^{1} \cdot R^{2} a_{i}^{2} \cdots R^{n_{i}} a_{i}^{n_{i}}
$$

( $R^{k}$ being $P$ or $V^{2}$ ), there is a natural way to understand the possible behaviours of their concurrent execution, by associating to each process a coordinate line in $\mathbb{R}^{n}$. The state of the system corresponds to a point in $\mathbb{R}^{n}$, whose $i$ th coordinate describes the state (or "local time") of the $i$ th processor.

${ }^{1}$ Of course this operation must be done "atomically", meaning that the semaphore itself must be handled in a mutually exclusive manner: this is done at the hardware level.

${ }^{2}$ Using E. W. Dijkstra's notation $P$ and $V[\mathbf{8}]$ for respectively acquiring and releasing a lock on a semaphore. 


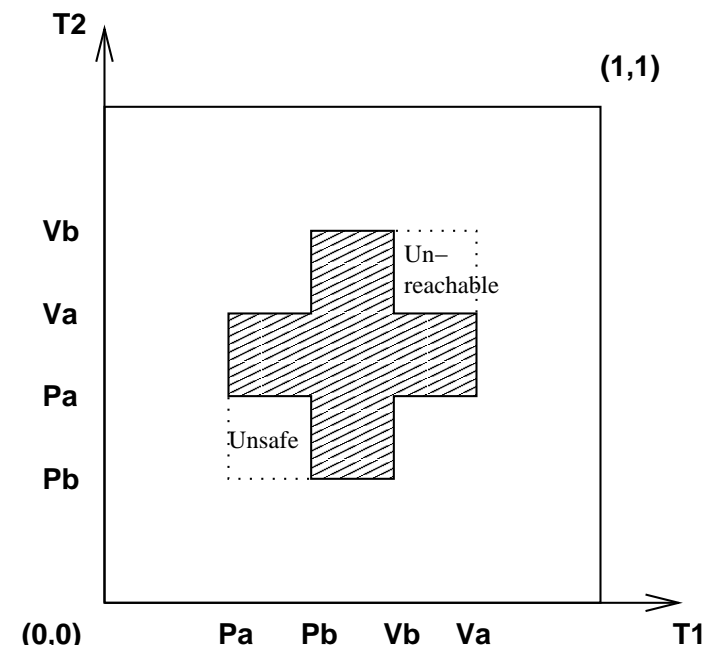

Figure 3: Example of a progress graph

Consider a system with finitely many processes running altogether. We assume that each process starts at (local time) 0 and finishes at (local time) 1 ; the $P$ and $V$ actions correspond to sequences of real numbers between 0 and 1 , which reflect the order of the $P$ 's and $V$ 's. The initial state is $(0, \ldots, 0)$ and the final state is $(1, \ldots, 1)$. An example consisting of the two processes $T_{1}=P a \cdot P b \cdot V b \cdot V a$ and $T_{2}=P b . P a . V a . V b$ gives rise to the two dimensional progress graph of Figure 3.

The shaded area represents states which are not allowed in any execution path, since they correspond to mutual exclusion. Such states constitute the forbidden area. An execution path is a path from the initial state $(0, \ldots, 0)$ to the final state $(1, \ldots, 1)$ avoiding the forbidden area and increasing in each coordinate - time cannot run backwards. This entails that paths reaching the states in the dashed square underneath the forbidden region, marked "unsafe" are deemed to deadlock, i.e. they cannot possibly reach the allowed terminal state which is $(1,1)$ here. Similarly, by reversing the direction of time, the states in the square above the forbidden region, marked "unreachable", cannot be reached from the initial state, which is $(0,0)$ here. Also notice that all terminating paths above the forbidden region are "equivalent" in some sense, given that they are all characterized by the fact that $T_{2}$ gets $a$ and $b$ before $T_{1}$ (as far as resources are concerned, we call this a schedule). Similarly, all paths below the forbidden region are characterized by the fact that $T_{1}$ gets $a$ and $b$ before $T_{2}$ does.

We end up the paragraph with the Swiss Flag example of Figure 3 described as a flow.

Let $n \geqslant 1$. Let $\mathbf{D}^{n}$ be the closed $n$-dimensional disk defined by the set of points $\left(x_{1}, \ldots, x_{n}\right)$ of $\mathbb{R}^{n}$ such that $x_{1}^{2}+\cdots+x_{n}^{2} \leqslant 1$ endowed with the topology induced by that of $\mathbb{R}^{n}$. Let $\mathbf{S}^{n-1}=\partial \mathbf{D}^{n}$ be the boundary of $\mathbf{D}^{n}$ for $n \geqslant 1$, that is the set of $\left(x_{1}, \ldots, x_{n}\right) \in \mathbf{D}^{n}$ such that $x_{1}^{2}+\cdots+x_{n}^{2}=1$. Notice that $\mathbf{S}^{0}$ is the discrete 


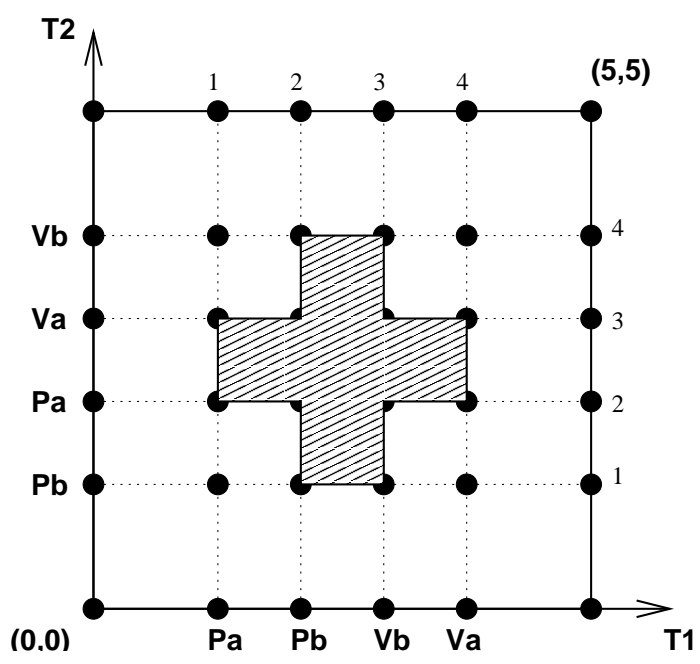

Figure 4: Example of a flow

two-point topological space $\{-1,+1\}$. Let $\mathbf{D}^{0}$ be the one-point topological space. Let $\mathbf{S}^{-1}=\varnothing$ be the empty set.

Consider the discrete set $S W^{0}=\{0,1,2,3,4,5\} \times\{0,1,2,3,4,5\}$. Let

$$
\begin{aligned}
\mathcal{S} & =\{((i, j),(i+1, j)) \text { for }(i, j) \in\{0, \ldots, 4\} \times\{0, \ldots, 5\}\} \\
& \cup\{((i, j),(i, j+1)) \text { for }(i, j) \in\{0, \ldots, 5\} \times\{0, \ldots, 4\}\} \\
& \backslash(\{((2,2),(2,3)),((2,2),(3,2)),((2,3),(3,3)),((3,2),(3,3))\})
\end{aligned}
$$

The flow $S W^{1}$ is obtained from $S W^{0}$ by attaching a copy of $\operatorname{Glob}\left(\mathbf{D}^{0}\right)$ to each pair $(x, y) \in \mathcal{S}$ with $x \in S W^{0}$ identified with 0 and $y \in S W^{0}$ identified with 1 . The flow $S W^{2}$ is obtained from $S W^{1}$ by attaching to each square $((i, j),(i+1, j+1))$ except $(i, j) \in\{(2,1),(1,2),(2,2),(3,2),(2,3)\}$ a globular cell $\mathrm{Glob}\left(\mathbf{D}^{1}\right)$ such that each execution path $((i, j),(i+1, j),(i+1, j+1))$ and $((i, j),(i, j+1),(i+1, j+1))$ is identified with one of the execution path of $\operatorname{Glob}\left(\mathbf{S}^{0}\right)$ (there is not a unique choice to do that). Let $S W=S W^{2}$ (cf. Figure 4 where the bold dots represent the points of the 0 -skeleton). The flow $S W$ represents the PV diagram of Figure 4.

\subsection{Limit and colimit in Flow}

Theorem 4.16. [2] [29] (Freyd's Adjoint Functor Theorem) Let $A$ and $X$ be locally small categories. Assume that $A$ is complete. Then a functor $G: A \longrightarrow X$ has a left adjoint if and only if it preserves all limits and satisfies the following "Solution Set Condition". For each object $x \in X$, there is a set of arrows $f_{i}: x \longrightarrow G a_{i}$ such that for every arrow $h: x \longrightarrow G a$ can be written as a composite $h=G t \circ f_{i}$ for some $i$ and some $t: a_{i} \longrightarrow a$.

Theorem 4.17. The category Flow is complete and cocomplete. In particular, a terminal object is the flow $\mathbf{1}$ having the discrete set $\{0, u\}$ as underlying topological 
space with 0 -skeleton $\{0\}$ and path space $\{u\}$. And an initial object is the unique flow $\varnothing$ having the empty set as underlying topological space.

Proof. Let $X: I \longrightarrow$ Flow be a functor from a small category $I$ to Flow. Let $Y$ be the flow defined as follows:

1. The 0 -skeleton $Y^{0}$ of $Y$ is defined as being the limit as sets $\varliminf_{I}\left(X(i)^{0}\right)$ equipped with the discrete topology.

2. Let $\alpha, \beta \in \varliminf_{I}\left(X(i)^{0}\right)$ and let $\alpha_{i}$ (resp. $\beta_{i}$ ) be the image of $\alpha(\beta)$ in $X(i)^{0}$. Then let $\mathbb{P}_{\alpha, \beta} Y:=\lim _{i} \mathbb{P}_{\alpha_{i}, \beta_{i}} X(i)$ where the limit is taken in Top.

3. For $\alpha, \beta, \gamma \in \lim _{I}\left(X(i)^{0}\right)$, let $\alpha_{i}$ (resp. $\left.\beta_{i}, \gamma_{i}\right)$ be the image of $\alpha$ (resp. $\beta, \gamma$ ) in $X(i)^{0}$. Then the composition map $*: \mathbb{P}_{\alpha, \beta} Y \times \mathbb{P}_{\beta, \gamma} Y \longrightarrow \mathbb{P}_{\alpha, \gamma} Y$ is taken as the limits of the $*_{i}: \mathbb{P}_{\alpha_{i}, \beta_{i}} X(i) \times \mathbb{P}_{\beta_{i}, \gamma_{i}} X(i) \longrightarrow \mathbb{P}_{\alpha_{i}, \gamma_{i}} X(i)$.

One does obtain a flow which is the $\operatorname{limit}_{\lim _{i \in I}} X(i)$. To prove that Flow is cocomplete, it suffices to prove that the constant diagram functor $\Delta_{I}$ from Flow to the category Flow ${ }^{I}$ of diagrams in Flow over the small category $I$ has a left adjoint using Theorem 4.16. The functor $\Delta_{I}$ commutes with limits. It suffices now to find a set of solutions. Consider a diagram $D$ of Flow $^{I}$. There is a class of solutions by taking all morphisms $f: D \rightarrow \Delta_{I} Y$ for $Y$ running over the category Flow and for $f$ running over the set of morphisms from $D$ to $\Delta_{I} Y$. Then one can suppose that $Y$ is the subflow generated by the image of $D$, so that the cardinal card $(Y)$ of $Y$ satisfies $\operatorname{card}(Y) \leqslant \aleph_{0} \times \operatorname{card}(D)$. Then it suffices to consider the set $\left\{Z_{i}, i \in I\right\}$ of isomorphism classes of flows whose underlying set is of cardinal less than $\aleph_{0} \times \operatorname{card}(D)$. Then $\operatorname{card}(I) \leqslant 2^{\left(\aleph_{0} \times \operatorname{card}(D)\right)^{5}}$. So $I$ is a set. Therefore $\bigcup_{i \in I}$ Flow $^{I}\left(D, \Delta_{I}\left(Z_{i}\right)\right)$ is a set as well. One has obtained a set of solutions.

\section{Morphisms of flows and colimits}

The aim of this section is the proof of the following theorem:

Theorem 5.1. (Theorem 5.10) Let FLOW $(X, Y)$ be the set of morphisms of flows from $X$ to $Y$ equipped with the Kelleyfication of the compact-open topology. Then the mapping

$$
(X, Y) \mapsto \mathbf{F L O W}(X, Y)
$$

induces a functor from $\mathbf{F l o w} \times$ Flow to Top which is contravariant with respect to $X$ and covariant with respect to $Y$. Moreover:

1. One has the natural homeomorphism

$$
\text { FLOW }\left(\underset{i}{\lim } X_{i}, Y\right) \cong \lim _{i} \operatorname{FLOW}\left(X_{i}, Y\right)
$$

for any colimit $\lim _{i} X_{i}$ in Flow. 
2. One has the natural homeomorphism

$$
\operatorname{FLOW}\left(X, \varliminf_{i} Y_{i}\right) \cong \varliminf_{i} \operatorname{FLOW}\left(X, Y_{i}\right)
$$

for any finite limit $\lim _{i} X_{i}$ in Flow.

\subsection{Non-contracting topological 1-category}

Definition 5.2. A non-contracting topological 1-category $X$ is a pair of compactly generated topological spaces $\left(X^{0}, \mathbb{P} X\right)$ together with continuous maps $s, t$ and * satisfying the same properties as in the definition of flow except that $X^{0}$ is not necessarily discrete. The corresponding category is denoted by $\mathbf{1 C a t}_{\mathbf{1}}^{\text {top }}$.

Definition 5.3. A non-contracting topological 1-category $X$ is achronal if $\mathbb{P} X=\varnothing$.

Theorem 5.4. The category $\mathbf{1} \mathbf{C a t}_{\mathbf{1}}^{\text {top }}$ is complete and cocomplete. The inclusion functor $\widetilde{\omega}:$ Flow $\longrightarrow \mathbf{1 C a t}_{\mathbf{1}}^{\text {top }}$ preserves finite limits.

Proof. Let $X: I \longrightarrow \mathbf{1 C a t}_{\mathbf{1}}^{\text {top }}$ be a functor from a small category $I$ to $\mathbf{1 C a t}_{\mathbf{1}}^{\text {top }}$. Then consider the topological 1-category $Y$ defined as follows:

1. Let $Y^{0}:=\lim _{i} X(i)^{0}$, the limit being taken in Top.

2. Let $\mathbb{P} Y:=\varliminf_{i} \mathbb{P} X(i)$, the limit being taken in Top.

3. Let $Y=Y^{0} \sqcup \mathbb{P} Y$ equipped with the source map, target map and composition law limits of the source maps, target maps and composition laws of the $X(i)$.

The 1-category $Y$ is clearly the $\operatorname{limit} \lim X$ in $\mathbf{1 C a t}_{\mathbf{1}}^{\text {top }}$. The cocompleteness of 1Cat $_{1}^{\text {top }}$ is then proved using the "solution set condition" recalled in Theorem 4.16 as in the proof of Theorem 4.17. A finite limit of discrete topological spaces is discrete. So to be able to conclude that the functor $\widetilde{\omega}$ preserves finite limits, it then suffices to compare the construction of limits in Flow in the proof of Theorem 4.17 and the construction of limits in $\mathbf{1 C a t}_{\mathbf{1}}^{\text {top }}$ in this proof.

Using the above constructions, one sees that the 0-skeleton functor

$$
(-)^{0}: \mathbf{1 C a t}_{1}^{\text {top }} \longrightarrow \text { Top }
$$

does commute with any limit. However the 0-skeleton functor $(-)^{0}:$ Flow $\longrightarrow$ Top only commutes with finite limits. On the contrary, both 0 -skeleton functors $(-)^{0}$ : $\mathbf{1 C a t}_{1}^{\text {top }} \longrightarrow$ Top and $(-)^{0}:$ Flow $\longrightarrow$ Top do commute with any colimit.

The functor $\widetilde{\omega}:$ Flow $\longrightarrow \mathbf{1 C a t}_{\mathbf{1}}^{\text {top }}$ does not preserve general limits. As counterexample, take the achronal 1-categories $\mathbb{Z} / p^{n} \mathbb{Z}$ equipped with the discrete topology and consider the tower of maps $\mathbb{Z} / p^{n+1} \mathbb{Z} \longrightarrow \mathbb{Z} / p^{n} \mathbb{Z}$ defined by $x \mapsto p . x$. Then the limit in Flow is the achronal flow having as 0-skeleton the set of $p$-adic integers $\mathbb{Z}_{p}$ and the limit in $\mathbf{1} \mathbf{C a t}_{\mathbf{1}}^{\text {top }}$ is a totally disconnected achronal 1-category.

Theorem 5.5. The inclusion functor $\widetilde{\omega}:$ Flow $\longrightarrow \mathbf{1 C a t}_{\mathbf{1}}^{\text {top }}$ has a right adjoint that will be denoted by $\widetilde{D}$. In particular, this implies that the canonical inclusion 
functor $\mathbf{F l o w} \longrightarrow \mathbf{1 C a t}_{\mathbf{1}}^{\text {top }}$ preserves colimits. Moreover, one has $\widetilde{D} \circ \widetilde{\omega}=\operatorname{Id}_{\text {Flow }}$ and

$$
\lim _{i} X_{i} \cong \lim _{i} \widetilde{D} \circ \widetilde{\omega}\left(X_{i}\right) \cong \widetilde{D}\left({\underset{\lim }{i}}_{i} \widetilde{\omega}\left(X_{i}\right)\right)
$$

If Set is the category of sets, then the forgetful functor $\omega:$ Top $\longrightarrow$ Set has a left adjoint: the functor $X \mapsto \operatorname{Dis}(X)$ which maps a set $X$ to the discrete space $\operatorname{Dis}(X)$. So

$$
\operatorname{Top}(D i s(X), Y) \cong \operatorname{Set}(X, \omega(Y)) .
$$

Proof. Let $\mathcal{C}$ be an object of $\mathbf{1} \mathbf{C a t}_{\mathbf{1}}^{\text {top }}$. Then:

- Let $\widetilde{D}(\mathcal{C})^{0}:=\mathcal{C}^{0}$ equipped with the discrete topology.

- If $(\alpha, \beta) \in \widetilde{D}(\mathcal{C})^{0} \times \widetilde{D}(\mathcal{C})^{0}$, let $\mathbb{P}_{\alpha, \beta} \widetilde{D}(\mathcal{C})$ be the subspace of $\mathbb{P C}$ of execution paths $x$ such that $s(x)=\alpha$ and $t(x)=\beta$ equipped with the Kelleyfication of the relative topology.

- Let $\mathbb{P} \widetilde{D}(\mathcal{C})=\bigsqcup_{(\alpha, \beta) \in \widetilde{D}(\mathcal{C})^{0} \times \widetilde{D}(\mathcal{C})^{0}} \mathbb{P}_{\alpha, \beta} \widetilde{D}(\mathcal{C})$ with an obvious definition of the source map $s$, the target map $t$ and the composition law $*$.

Let $f \in$ Flow $(X, \widetilde{D}(Y))$. Then the composite $X^{0} \longrightarrow \widetilde{D}(Y)^{0} \longrightarrow Y^{0}$ is continuous. And for any $\alpha, \beta \in X^{0}, \mathbb{P}_{\alpha, \beta} X \longrightarrow \mathbb{P}_{f(\alpha), f(\beta)} Y \longrightarrow Y$ is continuous as well. Reciprocally, a map $g \in \mathbf{1 C a t}_{\mathbf{1}}^{\text {top }}(\widetilde{\omega}(X), Y)$ provides $g^{0} \in \operatorname{Top}\left(\widetilde{\omega}(X)^{0}, Y^{0}\right) \cong$ Set $\left(\omega \circ \widetilde{\omega}(X)^{0}, \omega\left(Y^{0}\right)\right)$ since $\widetilde{\omega}(X)^{0}$ is a discrete space and provides a continuous map

$$
\mathbb{P} g \in \operatorname{Top}(\mathbb{P} \widetilde{\omega}(X), \mathbb{P} Y) \cong \operatorname{Top}\left(\bigsqcup_{(\alpha, \beta)} \mathbb{P}_{\alpha, \beta} X, \mathbb{P} Y\right) \longrightarrow \prod_{(\alpha, \beta)} \operatorname{Top}\left(\mathbb{P}_{\alpha, \beta} X, \mathbb{P} \widetilde{D} Y\right) .
$$

Hence the natural bijection

$$
\text { Flow }(X, \widetilde{D}(Y)) \cong \mathbf{1 C a t}_{\mathbf{1}}^{\text {top }}(\widetilde{\omega}(X), Y) .
$$

\subsection{Tensor product of non-contracting topological 1-categories}

The purpose of this section is the construction of a closed symmetric monoidal structure on $\mathbf{1 C a t}_{1}^{\text {top }}$. Let

$$
\mathbf{1 C A T}_{\mathbf{1}}^{\mathrm{top}}(Y, Z)
$$

be the set $\mathbf{1 C a t}_{\mathbf{1}}^{\text {top }}(Y, Z) \subset \mathbf{T O P}(Y, Z)$ equipped with the Kelleyfication of the relative topology induced by that of $\mathbf{T O P}(Y, Z)$.

Proposition 5.6. Let $X$ and $Y$ be two objects of $\mathbf{1 C a t}_{\mathbf{1}}^{\text {top }}$. There exists a unique structure of topological 1-category $X \otimes Y$ on the topological space $\left(X^{0} \sqcup \mathbb{P} X\right) \times$ $\left(Y^{0} \sqcup \mathbb{P} Y\right)$ such that

1. $(X \otimes Y)^{0}=X^{0} \times Y^{0}$. 
2. $\mathbb{P}(X \otimes Y)=(\mathbb{P} X \times \mathbb{P} X) \sqcup\left(X^{0} \times \mathbb{P} Y\right) \sqcup\left(\mathbb{P} X \times Y^{0}\right)$.

3. $s(x, y)=(s(x), s(y)), t(x, y)=(t(x), t(y)),(x, y) *(z, t)=(x * z, y * t)$.

Proof. Obvious.

Proposition 5.7. Let $X$ and $Y$ be two objects of $\mathbf{1 C a t}_{\mathbf{1}}^{\text {top }}$. Let $f$ and $g$ be two morphisms in $\mathbf{1 C a t}_{\mathbf{1}}^{\text {top }}$ from $\vec{I} \otimes X$ to $Y$. Let us suppose that for any $y \in Y$, $f(1 \otimes y)=g(0 \otimes y)$. Then for any $y \in X$, the following equality holds

$$
f([0,1] \otimes s(y)) * g([0,1] \otimes y)=f([0,1] \otimes y) * g([0,1] \otimes t(y))
$$

Denote the common value by $(f * g)([0,1] \otimes y)$. Let

$$
(f * g)(0 \otimes y)=f(0 \otimes y)
$$

and

$$
(f * g)(1 \otimes y)=g(1 \otimes y) .
$$

Then $f * g$ yields an element of $\mathbf{1 C a t}_{\mathbf{1}}^{\text {top }}(\vec{I} \otimes X, Y)$ and one has moreover $(f * g) *$ $h=f *(g * h)$. At last, this composition yields a continuous map from the fiber product

$$
\mathbf{1 C A T}_{\mathbf{1}}^{\text {top }}(\vec{I} \otimes X, Y) \times_{\mathbf{1 C A T}_{1}^{\text {top }}(X, Y)} \mathbf{1 C A T}_{\mathbf{1}}^{\text {top }}(\vec{I} \otimes X, Y)
$$

given by the inclusions $\{0\} \subset \vec{I}$ and $\{1\} \subset \vec{I}$ to $\mathbf{1 C A T}_{\mathbf{1}}^{\text {top }}(\vec{I} \otimes X, Y)$.

Proof. First of all, one has

$$
\begin{aligned}
& f([0,1] \otimes s(y)) * g([0,1] \otimes y) \\
& =f([0,1] \otimes s(y)) * g(0 \otimes y) * g([0,1] \otimes t(y)) \text { since } g \text { morphism of } \mathbf{1 C a t}_{\mathbf{1}}^{\text {top }} \\
& =f([0,1] \otimes s(y)) * f(1 \otimes y) * g([0,1] \otimes t(y)) \text { by hypothesis } \\
& =f([0,1] \otimes y) * g([0,1] \otimes t(y)) \quad \text { since } f \text { morphism of } \mathbf{1 C a t}_{\mathbf{1}}^{\text {top }}
\end{aligned}
$$

The equalities

$$
(f * g)(0 \otimes x * y)=(f * g)(0 \otimes x) *(f * g)(0 \otimes y)
$$

and

$$
(f * g)(1 \otimes x * y)=(f * g)(1 \otimes x) *(f * g)(1 \otimes y)
$$

are trivial. Because of the symmetries, it remains to check that

$$
(f * g)([0,1] \otimes x * y)=(f * g)(0 \otimes x) *(f * g)([0,1] \otimes y)
$$

to get $f * g \in \mathbf{1 C a t}_{\mathbf{1}}^{\text {top }}(\vec{I} \otimes X, Y)$. And one has

$$
\begin{aligned}
(f * g)([0,1] \otimes x * y) & =f([0,1] \otimes(x * y)) * g([0,1] \otimes t(x * y)) \\
& =f(0 \otimes x) * f([0,1] \otimes y) * g(0 \otimes t(y)) * g([0,1] \otimes t(y)) \\
& =f(0 \otimes x) * f([0,1] \otimes y) * f(1 \otimes t(y)) * g([0,1] \otimes t(y)) \\
& =f(0 \otimes x) * f([0,1] \otimes y) * g([0,1] \otimes t(y)) \\
& =(f * g)(0 \otimes x) *(f * g)([0,1] \otimes y) .
\end{aligned}
$$


At last, one has to check that $(f * g) * h=f *(g * h)$. Once again, the equalities

$$
((f * g) * h)(0 \otimes x)=(f *(g * h))(0 \otimes x)
$$

and

$$
((f * g) * h)(1 \otimes x)=(f *(g * h))(1 \otimes x)
$$

are trivial. And one has

$$
\begin{aligned}
((f * g) * h)([0,1] \otimes x) & =(f * g)([0,1] \otimes s(x)) * h([0,1] \otimes x) \\
& =f([0,1] \otimes s(x)) * g([0,1] \otimes s(x)) * h([0,1] \otimes x) \\
& =f([0,1] \otimes s(x)) *(g * h)([0,1] \otimes x) \\
& =(f *(g * h))([0,1] \otimes x) .
\end{aligned}
$$

The continuity of $*$ is due to the fact that we are working exclusively with compactly generated topological spaces.

Theorem 5.8. The tensor product of $\mathbf{1 C a t}_{\mathbf{1}}^{\text {top }}$ is a closed symmetric monoidal structure, that is there exists a bifunctor

$$
\left[\mathbf{C a t}_{1}^{\text {top }}\right]: \mathbf{1 C a t}_{1}^{\text {top }} \times 1 \mathrm{Cat}_{1}^{\text {top }} \longrightarrow \mathbf{1 C a t}_{1}^{\text {top }}
$$

contravariant with respect to the first argument and covariant with respect to the second argument such that one has the natural bijection of sets

$$
\mathbf{1 C a t}_{\mathbf{1}}^{\text {top }}(X \otimes Y, Z) \cong \mathbf{1 C a t}_{\mathbf{1}}^{\text {top }}\left(X,\left[\mathbf{1 C a t}_{\mathbf{1}}^{\text {top }}\right](Y, Z)\right)
$$

for any topological 1-categories $X, Y$ and $Z$.

Proof.

Construction of $\left[\mathbf{1 C a t}_{\mathbf{1}}^{\text {top }}\right](Y, Z)$

1. $\left[\mathbf{1 C a t}_{\mathbf{1}}^{\text {top }}\right](Y, Z)^{0}:=\mathbf{1 C A T}_{\mathbf{1}}^{\text {top }}(Y, Z)$

2. $\mathbb{P}\left[\mathbf{1 C a t}_{\mathbf{1}}^{\text {top }}\right](Y, Z):=\mathbf{1 C A T}_{\mathbf{1}}^{\text {top }}(\vec{I} \otimes Y, Z)$

3. the source map and target map are induced respectively by the morphisms $\{0\} \subset \vec{I}$ and $\{1\} \subset \vec{I}$

4. the composition law is defined by Proposition 5.7.

Construction of the set map $\Phi: \mathbf{1 C a t}_{\mathbf{1}}^{\text {top }}(X \otimes Y, Z) \longrightarrow$
$\mathbf{1 C a t}_{\mathbf{1}}^{\text {top }}\left(X,\left[\mathbf{1} \mathbf{C} \mathbf{a t}_{\mathbf{1}}^{\text {top }}\right](Y, Z)\right)\left(\right.$ with $\left.f \in \mathbf{1 C a t}_{\mathbf{1}}^{\text {top }}(X \otimes Y, Z)\right)$

1. for $x \in X^{0}, \Phi(f)(x)$ is the morphism of flows from $Y$ to $Z$ defined by

- $\Phi(f)(x)(y)=f(x \otimes y)$.

2. for $x \in \mathbb{P} X, \Phi(f)(x)$ is the morphism of flows from $\vec{I} \otimes Y$ to $Z$ defined by

- $\Phi(f)(x)(0 \otimes y)=f(s(x) \otimes y)$

- $\Phi(f)(x)(1 \otimes y)=f(t(x) \otimes y)$

- $\Phi(f)(x)([0,1] \otimes y)=f(x \otimes y)$. 


\begin{tabular}{lllll|} 
Construction of the & set & map & $\Psi$ & $:$ \\
$\mathbf{1 C a t}_{\mathbf{1}}^{\text {top }}\left(X,\left[\mathbf{1 C a t}_{\mathbf{1}}^{\text {top }}\right](Y, Z)\right)$ & $\longrightarrow$ & $\mathbf{1 C a t}_{\mathbf{1}}^{\text {top }}(X \otimes Y, Z)$ & (with $^{\text {top }}$ \\
$\left.g \in \mathbf{1 C a t}_{\mathbf{1}}^{\text {top }}\left(X,\left[\mathbf{1 C a t}_{\mathbf{1}}^{\text {top }}\right](Y, Z)\right)\right)$ & & & \\
\hline
\end{tabular}

1. $\Psi(g)\left(x_{0} \otimes y\right)=g\left(x_{0}\right)(y)$ for $\left(x_{0} \otimes y\right) \in X^{0} \times Y$

2. $\Psi(g)(x \otimes y)=g(x)([0,1] \otimes y)$ for $(x \otimes y) \in \mathbb{P} X \times Y$.

$\Phi(f)\left(x * x^{\prime}\right)=\Phi(f)(x) * \Phi(f)\left(x^{\prime}\right)\left(\right.$ with $\left.x, x^{\prime} \in \mathbb{P} X\right)$

1. $\Phi(f)\left(x * x^{\prime}\right)(0 \otimes y)=f(s(x) \otimes y)$

2. $\Phi(f)\left(x * x^{\prime}\right)([0,1] \otimes y)=f\left(\left(x * x^{\prime}\right) \otimes y\right)$

3. $\Phi(f)\left(x * x^{\prime}\right)(1 \otimes y)=f\left(t\left(x^{\prime}\right) \otimes y\right)$

$$
\Phi(f)(s(x))=s(\Phi(f)(x)) \text { and } \Phi(f)(t(x))=t(\Phi(f)(x))
$$

1. $\Phi(f)(s(x))=f(s(x) \otimes-)=s(\Phi(f)(x))$

2. $\Phi(f)(t(x))=f(t(x) \otimes-)=t(\Phi(f)(x))$.

$$
\begin{aligned}
& \Psi(g)\left(\left(x_{0} \otimes y\right) *\left(x_{0} \otimes y^{\prime}\right)\right)=\Psi(g)\left(x_{0} \otimes y\right) * \Psi(g)\left(x_{0} \otimes y^{\prime}\right) \text { (with } \\
& \left.g \in \text { Flow }\left(X,\left[\mathbf{1 C a t}_{\mathbf{1}}^{\text {top }}\right](Y, Z)\right), x_{0} \in X^{0}, y, y^{\prime} \in \mathbb{P} Y\right) \\
& \Psi(g)\left(\left(x_{0} \otimes y\right) *\left(x_{0} \otimes y^{\prime}\right)\right)=\Psi(g)\left(\left(x_{0} \otimes\left(y * y^{\prime}\right)\right)\right) \\
& =g\left(x_{0}\right)\left(y * y^{\prime}\right) \\
& =g\left(x_{0}\right)(y) * g\left(x_{0}\right)\left(y^{\prime}\right) \\
& =\Psi(g)\left(x_{0} \otimes y\right) * \Psi(g)\left(x_{0} \otimes y^{\prime}\right) \text {. }
\end{aligned}
$$

$\Psi(g)\left(\left(x_{0} \otimes y\right) *\left(x \otimes y^{\prime}\right)\right)=\Psi(g)\left(x_{0} \otimes y\right) * \Psi(g)\left(x \otimes y^{\prime}\right)($ with $g \in$ Flow $\left.\left(X,\left[\mathbf{1 C a t}_{\mathbf{1}}^{\text {top }}\right](Y, Z)\right), x_{0} \in X^{0}, x \in \mathbb{P} X, y, y^{\prime} \in \mathbb{P} Y\right)$

$$
\begin{aligned}
\Psi(g)\left(\left(x_{0} \otimes y\right) *\left(x \otimes y^{\prime}\right)\right) & =\Psi(g)\left(x \otimes\left(y * y^{\prime}\right)\right) \\
& =g(x)\left([0,1] \otimes\left(y * y^{\prime}\right)\right) \\
& =g(x)(0 \otimes y) * g(x)\left([0,1] \otimes y^{\prime}\right) \\
& =\Psi(g)\left(x_{0} \otimes y\right) * \Psi(g)\left(x \otimes y^{\prime}\right)
\end{aligned}
$$

$s(\Psi(g)(x \otimes y))=\Psi(g)(s(x) \otimes s(y))($ with $x \in \mathbb{P} X, y \in Y)$

$$
\begin{aligned}
s(\Psi(g)(x \otimes y)) & =s(g(x)([0,1] \otimes y)) \\
& =g(x)(s([0,1] \otimes y)) \\
& =g(x)(0 \otimes s(y)) \\
& =(s(g(x)))(s(y)) \\
& =(g(s(x)))(s(y)) \\
& =\Psi(g)(s(x) \otimes s(y))
\end{aligned}
$$




$$
\begin{aligned}
s\left(\Psi(g)\left(x_{0} \otimes y\right)\right)=\Psi(g)\left(x_{0} \otimes s(y)\right) & \left(\text { with } x_{0} \in X^{0}, y \in Y\right) \\
s\left(\Psi(g)\left(x_{0} \otimes y\right)\right) & =s\left(g\left(x_{0}\right)(y)\right) \\
= & g\left(x_{0}\right)(s(y)) \\
= & \Psi(g)\left(x_{0} \otimes s(y)\right)
\end{aligned}
$$

$$
\Phi \circ \Psi=\operatorname{Id}_{\mathbf{1} \mathbf{C a t}_{1}^{\mathrm{top}}\left(X,\left[\mathbf{C a t}_{\mathbf{1}}^{\mathrm{top}}\right](Y, Z)\right)}
$$

Let $x_{0} \in X^{0}$ and $y \in Y$. Then

$$
\Phi(\Psi(g))\left(x_{0}\right)(y)=\Psi(g)\left(x_{0} \otimes y\right)=g\left(x_{0}\right)(y)
$$

therefore $\Phi(\Psi(g))\left(x_{0}\right)=g\left(x_{0}\right)$. And for $x \in \mathbb{P} X$,

1. $\Phi(\Psi(g))(x)(0 \otimes y)=\Psi(g)(s(x) \otimes y)=g(s(x))(y)$

2. $\Phi(\Psi(g))(x)(1 \otimes y)=\Psi(g)(t(x) \otimes y)=g(t(x))(y)$

3. $\Phi(\Psi(g))(x)([0,1] \otimes y)=\Psi(g)(x \otimes y)=g(x)([0,1] \otimes y)$.

$$
\begin{aligned}
& \Psi \circ \Phi=\operatorname{Id}_{\mathbf{1 C a t}_{\mathbf{1}}^{\text {top }}(X \otimes Y, Z)} \\
& \text { With } f \in \mathbf{1 C a t}_{\mathbf{1}}^{\text {top }}(X \otimes Y, Z), x_{0} \in X^{0} \text { and } y \in Y, \text { one has } \\
& \Psi(\Phi(f))\left(x_{0} \otimes y\right)=\Phi(f)\left(x_{0}\right)(y)=f\left(x_{0} \otimes y\right)
\end{aligned}
$$

and for $x \in \mathbb{P} X$,

$$
\Psi(\Phi(f))(x \otimes y)=\Phi(f)(x)([0,1] \otimes y)=f(x \otimes y) .
$$

The continuity of $\Phi(f)$

1. The continuity of $\Phi(f)^{0}: X^{0} \longrightarrow \mathbf{1 C A T}_{\mathbf{1}}^{\text {top }}(Y, Z)$ because

$$
\Phi(f)^{0} \in \operatorname{Top}\left(X^{0}, \mathbf{T O P}(Y, Z)\right) \cong \operatorname{Top}\left(X^{0} \times Y, Z\right) .
$$

2. The continuity of $\mathbb{P} \Phi(f): \mathbb{P} X \longrightarrow \mathbf{1 C A T}_{\mathbf{1}}^{\text {top }}(\vec{I} \otimes Y, Z)$ because

$$
\mathbb{P} \Phi(f) \in \operatorname{Top}(\mathbb{P} X, \mathbf{T O P}(\vec{I} \times Y, Z)) \cong \operatorname{Top}(\mathbb{P} X \times \vec{I} \times Y, Z) .
$$

The continuity of $\Psi(g)$

The continuity of $\Psi(g)$ comes again from the canonical bijections of sets

$$
\operatorname{Top}\left(X^{0}, \boldsymbol{T O P}(Y, Z)\right) \cong \operatorname{Top}\left(X^{0} \times Y, Z\right)
$$

and

$$
\operatorname{Top}(\mathbb{P} X, \boldsymbol{T O P}(\vec{I} \times Y, Z)) \cong \operatorname{Top}(\mathbb{P} X \times \vec{I} \times Y, Z)
$$

and also from the fact that the underlying topological space of a given 1-category $X$ is homeomorphic to the disjoint sum of topological spaces $X^{0} \sqcup \mathbb{P} X$. This completes the proof.

Corollary 5.9. Let $X$ and $Y$ be two topological 1-categories. Then one has the homeomorphisms

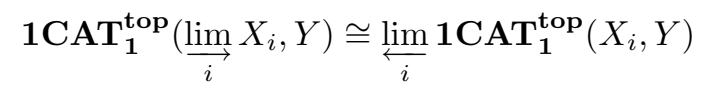


and

$$
\mathbf{1 C A T}_{\mathbf{1}}^{\mathbf{t o p}}\left(X,{\underset{\leftarrow}{i}}_{\lim _{i}} Y_{i}\right) \cong \varliminf_{i} \lim _{\mathbf{C}} \mathbf{C A T}_{\mathbf{1}}^{\mathbf{t o p}}\left(X, Y_{i}\right)
$$

for any colimit ${\underline{\varliminf_{i}}}_{i} X_{i}$ and any limit $\varliminf_{i} Y_{i}$ in $\mathbf{1 C a t}_{\mathbf{1}}^{\text {top }}$.

In both following calculations, one uses the fact that the following natural homeomorphism holds in $\mathbf{1 C a t}_{\mathbf{1}}^{\text {top }}:\left(\varliminf_{i} X_{i}\right)^{0} \cong \varliminf_{i}\left(X_{i}^{0}\right)$. The latter homeomorphism may be false in Flow since the 0 -skeleton is always discrete in the latter category.

Proof. One has:

$$
\begin{aligned}
& \mathbf{1 C A T}_{\mathbf{1}}^{\mathbf{t o p}}\left(\underset{i}{\lim } X_{i}, Y\right) \cong\left(\left[\mathbf{1 C a t}_{\mathbf{1}}^{\mathbf{t o p}}\right]\left(\underset{i}{\lim } X_{i}, Y\right)\right)^{0} \\
& \cong\left(\varliminf_{i}\left[\mathbf{C} \mathbf{C a t}_{\mathbf{1}}^{\text {top }}\right]\left(X_{i}, Y\right)\right)^{0} \\
& \cong \lim _{i}\left(\left[\mathbf{1 C a t}_{\mathbf{1}}^{\text {top }}\right]\left(X_{i}, Y\right)\right)^{0} \\
& \cong \overleftarrow{\lim }_{i} \mathbf{C C A T}_{\mathbf{1}}^{\mathbf{t o p}}\left(X_{i}, Y\right)
\end{aligned}
$$

and

$$
\begin{aligned}
& \mathbf{1 C A T}_{\mathbf{1}}^{\text {top }}\left(X, \overleftarrow{i}_{i} Y_{i}\right) \cong\left(\left[\mathbf{C a t}_{\mathbf{1}}^{\text {top }}\right]\left(X, \stackrel{\lim }{i}_{i} Y_{i}\right)\right)^{0} \\
& \cong\left(\varliminf_{i}^{\lim }\left[\mathbf{C a t}_{\mathbf{1}}^{\mathbf{t o p}}\right]\left(X, Y_{i}\right)\right)^{0} \\
& \cong \lim _{i}\left(\left[\mathbf{1 C a t}_{\mathbf{1}}^{\text {top }}\right]\left(X, Y_{i}\right)\right)^{0} \\
& \cong \varliminf_{i} \mathbf{l i m}_{\mathbf{1}}^{\mathbf{t o p}}\left(X, Y_{i}\right) \text {. }
\end{aligned}
$$

\subsection{Important consequence for the category of flows}

As an application of the preceding results, one proves the following crucial theorem:

Theorem 5.10. Let FLOW $(X, Y)$ be the set of morphisms of flows from $X$ to $Y$ equipped with the Kelleyfication of the compact-open topology. Then the mapping

$$
(X, Y) \mapsto \text { FLOW }(X, Y)
$$

induces a functor from Flow $\times$ Flow to Top which is contravariant with respect to $X$ and covariant with respect to $Y$. Moreover: 
1. One has the natural homeomorphism

$$
\text { FLOW }\left(\underset{i}{\lim } X_{i}, Y\right) \cong \varliminf_{i} \operatorname{FLOW}\left(X_{i}, Y\right)
$$

for any colimit $\lim _{i} X_{i}$ in Flow .

2. One has the natural homeomorphism

$$
\operatorname{FLOW}\left(X, \varliminf_{i}^{\lim _{i}} Y_{i}\right) \cong \varliminf_{i} \operatorname{FLOW}\left(X, Y_{i}\right)
$$

for any finite limit $\lim _{i} X_{i}$ in Flow.

The functor $\mathbf{F L O W}(X,-)$ cannot commute with any limit. Indeed, with $X=$ $\{0\}$, one has $\mathbf{F L O W}(X, Y) \cong Y^{0}$ as space. However, a limit of a diagram of discrete topological space may be totally disconnected without being discrete.

This is the reason why we make the distinction between the set of morphisms Flow $(X, Y)$ from a flow $X$ to a flow $Y$ and the space of morphisms FLOW $(X, Y)$ from a flow $X$ to a flow $Y$.

Proof. Since $\widetilde{\omega}$ preserves colimits by Theorem 5.5, one has:

$$
\begin{aligned}
& \mathbf{F L O W}\left(\underset{i}{\lim _{\vec{i}}} X_{i}, Y\right) \cong \mathbf{1 C A T}_{\mathbf{1}}^{\mathbf{t o p}}\left(\widetilde{\omega}\left(\underset{i}{\lim _{\vec{i}}} X_{i}\right), \widetilde{\omega}(Y)\right) \\
& \cong \mathbf{1 C A T}_{\mathbf{1}}^{\mathbf{t o p}}\left(\underset{i}{\underset{i}{\lim } \widetilde{\omega}}\left(X_{i}\right), \widetilde{\omega}(Y)\right) \\
& \cong \lim _{i} \mathbf{C A A T}_{\mathbf{1}}^{\mathbf{t o p}}\left(\widetilde{\omega}\left(X_{i}\right), \widetilde{\omega}(Y)\right) \\
& \cong \varliminf_{i} \mathbf{F L O W}\left(X_{i}, Y\right)
\end{aligned}
$$

Since $\widetilde{\omega}$ preserves finite limits by Theorem 5.4, one has:

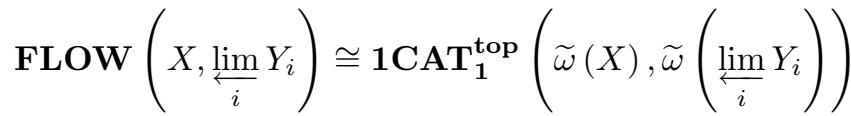

$$
\begin{aligned}
& \cong \mathbf{C A A T}_{\mathbf{1}}^{\text {top }}\left(\widetilde{\omega}(X), \stackrel{\lim }{i}_{i} \widetilde{\omega}\left(Y_{i}\right)\right) \\
& \cong \lim _{i} \mathbf{1 C A T}_{\mathbf{1}}^{\text {top }}\left(\widetilde{\omega}(X), \widetilde{\omega}\left(Y_{i}\right)\right) \\
& \cong \lim _{i} \mathbf{F L O W}\left(X, Y_{i}\right) \text {. }
\end{aligned}
$$

One does not need actually the previous machinery of tensor product of 1categories to prove the isomorphism of topological spaces

$$
\text { FLOW }\left(X,{\underset{i}{i}}_{\lim _{i}} Y_{i}\right) \cong{\underset{\varliminf}{i}}_{\lim _{i}} \mathbf{F L O W}\left(X, Y_{i}\right)
$$


for any finite limit $\lim _{i} Y_{i}$ of Flow. Indeed one sees that the forgetful functor $X \mapsto$ $X^{0} \sqcup \mathbb{P} X$ from Flow to Top induces the inclusion of topological spaces

$$
\text { FLOW } \left.\left.\left.X,{\underset{i}{i}}_{i}^{\lim } Y_{i}\right) \subset \text { TOP } X^{0},{\underset{i}{i}}_{i}^{\lim } Y_{i}^{0}\right) \times \prod_{(\alpha, \beta) \in X^{0} \times X^{0}} \text { TOP } \mathbb{P}_{\alpha, \beta} X,{\underset{i}{i}}_{\lim } \mathbb{P}_{\alpha_{i}, \beta_{i}} Y_{i}\right)
$$

where $\alpha_{i}$ (resp. $\beta_{i}$ ) is the image of $\alpha$ (resp. $\beta$ ) by the composite $X^{0} \longrightarrow \lim _{i} Y_{i}^{0} \longrightarrow$ $Y_{i}^{0}$. Since the right member of the above inclusion is isomorphic to

$$
\varliminf_{i}\left(\operatorname{TOP}\left(X^{0}, Y_{i}^{0}\right) \times \prod_{(\alpha, \beta) \in X^{0} \times X^{0}} \operatorname{TOP}\left(\mathbb{P}_{\alpha, \beta} X, \mathbb{P}_{\alpha_{i}, \beta_{i}} Y_{i}\right)\right)
$$

then the conclusion follows.

On the contrary, the forgetful functor $X \mapsto X^{0} \sqcup \mathbb{P} X$ from Flow to Top does not commute at all with colimits, even the finite ones, because colimits in 1-categories may create execution paths. So the tensor product of 1-categories seems to be required to establish the other homeomorphism.

\section{Flow as a canonical colimit of globes and points}

In the sequel, one will implicitely use the category $\mathcal{D}$ (Flow) of diagrams of flows. The objects are the functor $D: I \longrightarrow$ Flow where $I$ is a small category. A morphism from a diagram $D: I \longrightarrow$ Flow to a diagram $E: J \longrightarrow$ Flow is a functor $\phi: I \longrightarrow J$ together with a natural transformation $\mu: D \longrightarrow E \circ \phi$. A morphism of diagram $(\phi, \mu): D \longrightarrow E$ gives rise to a morphism of flows $\lim D \longrightarrow \lim E$. Since Flow is complete and cocomplete, then $\mathcal{D}$ (Flow) is complete and cocomplete as well $[\mathbf{2 4}]$.

In this section, we prove that any flow is the colimit in a canonical way of globes and points. This technical tool will be used in the sequel of the paper.

Theorem 6.1. Any flow is the colimit in Flow of points and globes in a canonical way, i.e. there exists for any flow $X$ a diagram $\mathbb{D}(X)$ of flows containing only points, globes and concatenations of globes such that the mapping $X \mapsto \mathbb{D}(X)$ is functorial and such that $X \cong \lim \mathbb{D}(X)$ in a canonical way.

Proof. Let $X$ be a flow and let $\alpha, \beta$ and $\gamma$ be three points (not necessarily distinct) of its 0 -skeleton. Consider the diagram of Figure 5 where the map

$$
\operatorname{Glob}\left(\mathbb{P}_{\alpha, \beta} X \times \mathbb{P}_{\beta, \gamma} X\right) \longrightarrow \operatorname{Glob}\left(\mathbb{P}_{\alpha, \beta} X\right) * \operatorname{Glob}\left(\mathbb{P}_{\beta, \gamma} X\right)
$$

is induced by the map $(x, y) \mapsto x * y$ (where $*$ is the free concatenation) and where the map

$$
\operatorname{Glob}\left(\mathbb{P}_{\alpha, \beta} X \times \mathbb{P}_{\beta, \gamma} X\right) \longrightarrow \operatorname{Glob}\left(\mathbb{P}_{\alpha, \gamma} X\right)
$$

is induced by the composition law of $X$. Then consider the diagram $\mathbb{D}(X)$ obtained by concatening all diagrams as that of Figure 5 . It is constructed as follows:

- the underlying small category $\mathcal{I}(X)$ of $\mathbb{D}(X)$ is the free category generated by the set of objects $X^{0} \cup X^{0} \times X^{0} \cup X^{0} \times X^{0} \times X^{0} \times\{0,1\}$ and by the arrows 
$-i_{1}^{\alpha, \beta}: \alpha \longrightarrow(\alpha, \beta)$ and $i_{2}^{\alpha, \beta}: \beta \longrightarrow(\alpha, \beta)$

$-r^{\alpha, \beta, \gamma}:(\alpha, \beta, \gamma, 0) \longrightarrow(\alpha, \beta, \gamma, 1)$ and $p^{\alpha, \beta, \gamma}:(\alpha, \beta, \gamma, 0) \longrightarrow(\alpha, \gamma)$

$-j_{1}^{\alpha, \beta, \gamma}: \alpha \longrightarrow(\alpha, \beta, \gamma, 0)$ and $j_{3}^{\alpha, \beta, \gamma}: \gamma \longrightarrow(\alpha, \beta, \gamma, 0)$

$-k_{1}^{\alpha, \beta, \gamma}: \alpha \longrightarrow(\alpha, \beta, \gamma, 1), k_{2}^{\alpha, \beta, \gamma}: \beta \longrightarrow(\alpha, \beta, \gamma, 1)$ and $k_{3}^{\alpha, \beta, \gamma}: \gamma \longrightarrow$ $(\alpha, \beta, \gamma, 1)$

$-h_{1}^{\alpha, \beta, \gamma}:(\alpha, \beta) \longrightarrow(\alpha, \beta, \gamma, 1)$ and $h_{3}^{\alpha, \beta, \gamma}:(\beta, \gamma) \longrightarrow(\alpha, \beta, \gamma, 1)$

- $\mathbb{D}(X)(\alpha)=\{\alpha\}, \mathbb{D}(X)(\alpha, \beta)=\operatorname{Glob}\left(\mathbb{P}_{\alpha, \beta} X\right)$

- $\mathbb{D}(X)(\alpha, \beta, \gamma, 0)=\operatorname{Glob}\left(\mathbb{P}_{\alpha \beta} X \times \mathbb{P}_{\beta, \gamma} X\right)$

- $\mathbb{D}(X)(\alpha, \beta, \gamma, 1)=\operatorname{Glob}\left(\mathbb{P}_{\alpha \beta} X\right) * \operatorname{Glob}\left(\mathbb{P}_{\beta, \gamma} X\right)$

- $\mathbb{D}(X)\left(i_{1}^{\alpha, \beta}\right)$ is the canonical inclusion

$$
\{\alpha\} \longrightarrow \operatorname{Glob}\left(\mathbb{P}_{\alpha, \beta} X\right)
$$

- $\mathbb{D}(X)\left(i_{2}^{\alpha, \beta}\right)$ is the canonical inclusion

$$
\{\beta\} \longrightarrow \operatorname{Glob}\left(\mathbb{P}_{\alpha, \beta} X\right)
$$

- $\mathbb{D}(X)\left(r^{\alpha, \beta, \gamma}\right)$ is the canonical projection

$$
\operatorname{Glob}\left(\mathbb{P}_{\alpha, \beta} X \times \mathbb{P}_{\beta, \gamma} X\right) \longrightarrow \operatorname{Glob}\left(\mathbb{P}_{\alpha, \beta} X\right) * \operatorname{Glob}\left(\mathbb{P}_{\beta, \gamma} X\right)
$$

sending $(x, y)$ to $x * y$.

- $\mathbb{D}(X)\left(p^{\alpha, \beta, \gamma}\right): \operatorname{Glob}\left(\mathbb{P}_{\alpha \beta} X \times \mathbb{P}_{\beta, \gamma} X\right) \longrightarrow \operatorname{Glob}\left(\mathbb{P}_{\alpha, \gamma} X\right)$ is the morphism induced by the composition law of $X$

- $\mathbb{D}(X)\left(j_{1}^{\alpha, \beta, \gamma}\right)\left(\right.$ resp. $\left.\mathbb{D}(X)\left(j_{3}^{\alpha, \beta, \gamma}\right)\right)$ is the canonical inclusion from $\{\alpha\}$ (resp. $\{\gamma\})$ to

$$
\operatorname{Glob}\left(\mathbb{P}_{\alpha, \beta} X \times \mathbb{P}_{\beta, \gamma} X\right)
$$

- $\mathbb{D}(X)\left(k_{1}^{\alpha, \beta, \gamma}\right)$ (resp. $\left.\mathbb{D}(X)\left(k_{2}^{\alpha, \beta, \gamma}\right), \mathbb{D}(X)\left(k_{3}^{\alpha, \beta, \gamma}\right)\right)$ is the canonical inclusion from $\{\alpha\}$ (resp. $\{\beta\},\{\gamma\}$ ) to

$$
\operatorname{Glob}\left(\mathbb{P}_{\alpha \beta} X\right) * \operatorname{Glob}\left(\mathbb{P}_{\beta, \gamma} X\right)
$$

- $\mathbb{D}(X)\left(h_{1}^{\alpha, \beta, \gamma}\right)$ is the canonical inclusion

$$
\operatorname{Glob}\left(\mathbb{P}_{\alpha, \beta} X\right) \longrightarrow \operatorname{Glob}\left(\mathbb{P}_{\alpha, \beta} X\right) * \operatorname{Glob}\left(\mathbb{P}_{\beta, \gamma} X\right)
$$

- $\mathbb{D}(X)\left(h_{3}^{\alpha, \beta, \gamma}\right)$ is the canonical inclusion

$$
\operatorname{Glob}\left(\mathbb{P}_{\beta, \gamma} X\right) \longrightarrow \operatorname{Glob}\left(\mathbb{P}_{\alpha, \beta} X\right) * \operatorname{Glob}\left(\mathbb{P}_{\beta, \gamma} X\right)
$$

Let $T$ be a flow. Let $f: \underline{\lim }_{i \in \mathcal{I}(X)} \mathbb{D}(X)(i) \longrightarrow T$ be a morphism of flows. Notice that all morphisms of flows in the diagram $\mathbb{D}(X)$ are source and target preserving. So $f$ yields a well-defined set map $g^{0}$ from $Y^{0}=X^{0}$ to $T^{0}$. Moreover the morphism $f$ yields a continuous map $f_{\alpha, \beta}: \mathbb{P}_{\alpha, \beta} X \longrightarrow \mathbb{P}_{f(\alpha), f(\beta)} T$ and for any $(\alpha, \beta, \gamma) \in$ $Y^{0} \times Y^{0} \times Y^{0}$, a continuous map

$$
f_{\alpha, \beta, \gamma, 0}: \mathbb{P}_{\alpha, \beta} X \times \mathbb{P}_{\beta, \gamma} X \longrightarrow \mathbb{P}_{f(\alpha), f(\gamma)} T
$$




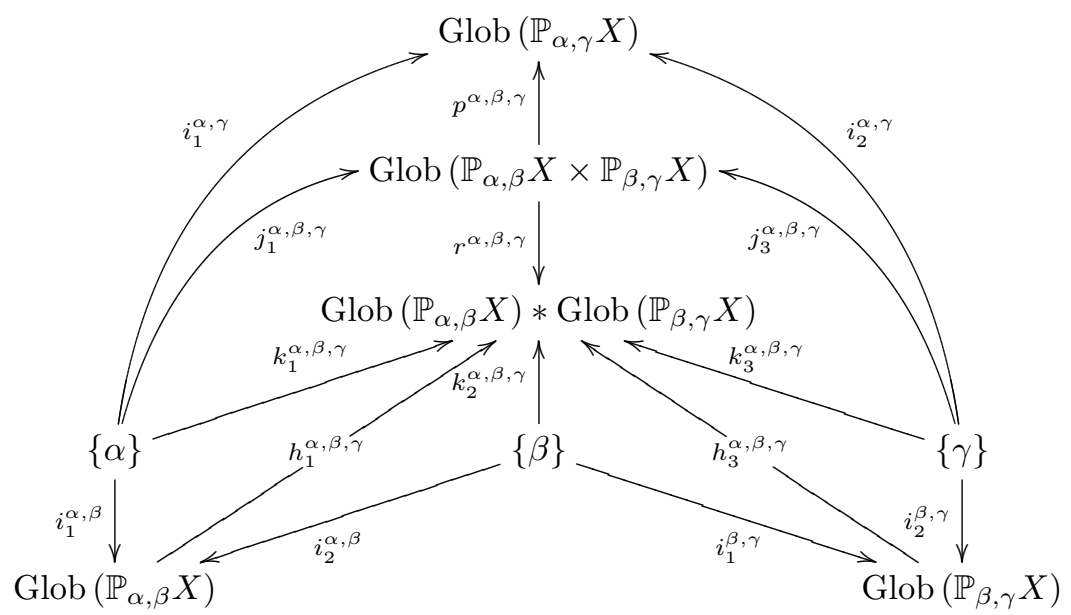

Figure 5: The flow $X$ as a colimit of globes and points

and another continuous map

$f_{\alpha, \beta, \gamma, 1}: \mathbb{P}_{\alpha, \beta} X \cup \mathbb{P}_{\beta, \gamma} X \cup \mathbb{P}_{\alpha, \beta} X \times \mathbb{P}_{\beta, \gamma} X \longrightarrow \mathbb{P}_{f(\alpha), f(\beta)} T \cup \mathbb{P}_{f(\beta), f(\gamma)} T \cup \mathbb{P}_{f(\alpha), f(\gamma)} T$

which satisfy various commutativity conditions. In particular all these maps define a unique continuous map $g_{\alpha, \beta}: \mathbb{P}_{\alpha, \beta} X \longrightarrow \mathbb{P}_{f(\alpha), f(\beta)} T$ thanks to $h_{1}^{\alpha, \beta, \gamma}$ and $h_{3}^{\alpha, \beta, \gamma}$ (these latter being inclusions). For $x \in \mathbb{P}_{\alpha, \beta} X$ and $y \in \mathbb{P}_{\beta, \gamma} X$, one has:

$$
\begin{aligned}
g_{\alpha, \gamma}(x * y) & =g_{\alpha, \gamma}\left(p^{\alpha, \beta, \gamma}(x, y)\right) \\
& =f_{\alpha, \beta, \gamma, 0}(x, y) \\
& =f_{\alpha, \beta, \gamma, 1}\left(r^{\alpha, \beta, \gamma}(x, y)\right) \\
& =f_{\alpha, \beta, \gamma, 1}(x * y) \\
& =f_{\alpha, \beta, 1}(x) * f_{\beta, \gamma, 1}(y) \text { since } f \text { morphism of flows ! } \\
& =f_{\alpha, \beta, \gamma, 1}\left(h_{1}^{\alpha, \beta, \gamma}(x)\right) * f_{\alpha, \beta, \gamma, 1}\left(h_{3}^{\alpha, \beta, \gamma}(y)\right) \\
& =f_{\alpha, \beta}(x) * f_{\beta, \gamma}(y) \\
& =g_{\alpha, \beta}(x) * g_{\beta, \gamma}(y)
\end{aligned}
$$

So $g$ yields a well-defined morphism of flows from $X$ to $T$. Conversely from a morphism of flows from $X$ to $T$, one can construct a morphism of flows from $\lim _{i \in \mathcal{I}(X)} \mathbb{D}(X)(i)$ to $T$. So one has the natural bijection of sets

$$
\text { Flow }\left(\lim _{i \in \mathcal{I}(X)} \mathbb{D}(X)(i), T\right) \cong \text { Flow }(X, T)
$$

Hence by Yoneda, the flow $X$ is the colimit of this diagram and moreover everything is canonical. The functoriality of $\mathbb{D}$ is obvious. 
Corollary 6.2. Let $P(X)$ be a statement depending on a flow $X$ and satisfying the following property: if $D: \mathcal{I} \longrightarrow$ Flow is a diagram of flows such that for any object $i$ of $\mathcal{I}, P(D(i))$ holds, then $P(\stackrel{\lim }{\longrightarrow})$ holds. Then the following assertions are equivalent:

(i) The statement $P(X)$ holds for any flow $X$ of Flow.

(ii) The statements $P(\{*\})$ and $P($ Glob $(Z))$ hold for any object $Z$ of Top.

Proof. The implication $(i) \Longrightarrow(i i)$ is obvious. Conversely if $(i i)$ holds, then

$$
P\left(\operatorname{Glob}\left(Z_{1}\right) * \operatorname{Glob}\left(Z_{2}\right)\right)
$$

holds for any topological spaces $Z_{1}$ and $Z_{2}$ since $\operatorname{Glob}\left(Z_{1}\right) * \operatorname{Glob}\left(Z_{2}\right)$ is the colimit of the diagram of flows

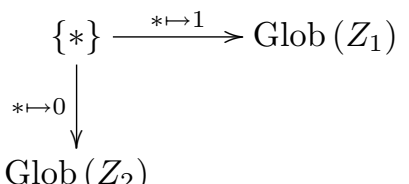

containing only points and globes. The proof is complete with Theorem 6.1.

\section{S-homotopy in Flow}

7.1. Synchronized morphism of flows

Definition 7.1. A morphism of flows $f: X \longrightarrow Y$ is said synchronized if and only if it induces a bijection of sets between the 0 -skeleton of $X$ and the 0 -skeleton of $Y$.

\subsection{S-homotopy of flows}

We mimick here the definition of the S-homotopy relation for globular complexes $[20]$.

Definition 7.2. Let $f$ and $g$ be two morphisms of flows from $X$ to $Y$. Then $f$ and $g$ are S-homotopic or S-homotopy equivalent if there exists a continuous map $H:[0,1] \times X \longrightarrow Y$ such that, with $H(u,-)=H_{u}$, for any $u \in[0,1], H_{u}$ is a morphism of flows from $X$ to $Y$ with $H_{0}=f$ and $H_{1}=g$. In particular, this implies that $f$ and $g$ coincide on the 0 -skeleton $X^{0}$ of $X$ and that for any $x_{0} \in X^{0}$, for any $u \in[0,1], f\left(x_{0}\right)=H\left(u, x_{0}\right)=g\left(x_{0}\right)$. This situation is denoted by $f \sim_{S} g$. This defines an equivalence relation on the set Flow $(X, Y)$.

Following Proposition 4.15, one then obtains the natural definition

Definition 7.3. Two elements of the path space $\mathbb{P} X$ of a flow $X$ are said Shomotopic if the corresponding morphisms of flows from $\vec{I}$ to $X$ are S-homotopy equivalent.

Definition 7.4. Two flows $X$ and $Y$ are S-homotopic or S-homotopy equivalent if there exists two morphisms $f: X \longrightarrow Y$ and $g: Y \longrightarrow X$ such that $f \circ g \sim_{S} \operatorname{Id}_{Y}$ and $g \circ f \sim_{S} \operatorname{Id}_{X}$. The maps $f$ and $g$ are called (reciprocal) S-homotopy equivalences. The 
S-homotopy relation is obviously an equivalence relation. We say that the mapping $g$ is a S-homotopic inverse of $f$.

Because of the discreteness of the 0-skeleton of any flow, a S-homotopy equivalence is necessarily synchronized.

Proposition 7.5. Let $f$ and $g$ be two morphisms of flows from $X$ to $Y$. Then $f$ and $g$ are $S$-homotopic if and only if there exists a continuous map

$$
h \in \operatorname{Top}([0,1], \mathbf{F L O W}(X, Y))
$$

such that $h(0)=f$ and $h(1)=g$.

Proof. Let $H:[0,1] \times X \longrightarrow Y$ be the S-homotopy from $f$ to $g$. Then $H$ provides an element of $\mathbf{T o p}([0,1], \mathbf{T O P}(X, Y))$ which is by definition of a S-homotopy also an element of Top $([0,1]$, FLOW $(X, Y))$. Conversely, an element $h$ of

$$
\operatorname{Top}([0,1], \mathbf{F L O W}(X, Y))
$$

yields an element of $\mathbf{T o p}([0,1]$, $\mathbf{T O P}(X, Y)) \cong \operatorname{Top}([0,1] \times X, Y)$ which is by construction a S-homotopy from $f$ to $g$.

\subsection{Pairing $\otimes$ between a topological space and a flow}

Notation 7.6. Let $U$ be a topological space. Let $X$ be a flow. The flow $\{U, X\}_{S}$ is defined as follows:

1. The 0-skeleton of $\{U, X\}_{S}$ is $X^{0}$.

2. For $\alpha, \beta \in X^{0}$, the topological space $\mathbb{P}_{\alpha, \beta}\{U, X\}_{S}$ is $\mathbf{T O P}\left(U, \mathbb{P}_{\alpha, \beta} X\right)$.

3. For $\alpha, \beta, \gamma \in X^{0}$, the composition law

$$
*: \mathbb{P}_{\alpha, \beta}\{U, X\}_{S} \times \mathbb{P}_{\beta, \gamma}\{U, X\}_{S} \longrightarrow \mathbb{P}_{\alpha, \gamma}\{U, X\}_{S}
$$

is the composite

$$
\mathbb{P}_{\alpha, \beta}\{U, X\}_{S} \times \mathbb{P}_{\beta, \gamma}\{U, X\}_{S} \cong \mathbf{T O P}\left(U, \mathbb{P}_{\alpha, \beta} X \times \mathbb{P}_{\beta, \gamma} X\right) \longrightarrow \mathbf{T O P}\left(U, \mathbb{P}_{\alpha, \gamma} X\right)
$$

induced by the composition law of $X$.

If $U=\varnothing$ is the empty set, then $\{\varnothing, Y\}_{S}$ is the flow having the same 0 -skeleton as $Y$ and exactly one non-constant execution path between two points of $Y^{0}$.

Theorem 7.7. Let $U$ be a topological space. The mapping $Y \mapsto\{U, Y\}_{S}$ yields a functor from Flow to itself. Moreover one has

1. one has the natural isomorphism of flows $\left\{U, \lim _{i} X_{i}\right\}_{S} \cong \lim _{i}\left\{U, X_{i}\right\}_{S}$

2. if $Y=Y^{0}$, then $\{U, Y\}_{S}=Y$

3. if $U$ and $V$ are two topological spaces, then $\{U \times V, Y\}_{S} \cong\left\{U,\{V, Y\}_{S}\right\}_{S}$.

Proof. The functoriality of $\{U,-\}_{S}$ is obvious. Following the proof of Theorem 4.17, it is clear that the functor $\{U,-\}_{S}$ does preserve limits in Flow. By definition,

$$
\{U \times V, Y\}_{S}^{0} \cong\left\{U,\{V, Y\}_{S}\right\}_{S}^{0} \cong Y^{0}
$$


and for $\alpha, \beta \in Y^{0}$, one has

$$
\mathbb{P}_{\alpha, \beta}\{U \times V, Y\}_{S}=\mathbf{T O P}\left(U \times V, \mathbb{P}_{\alpha, \beta} Y\right)
$$

and

$$
\mathbb{P}_{\alpha, \beta}\left\{U,\{V, Y\}_{S}\right\}_{S}=\mathbf{T O P}\left(U, \mathbf{T O P}\left(V, \mathbb{P}_{\alpha, \beta} Y\right)\right) .
$$

Therefore $\{U \times V, Y\}_{S} \cong\left\{U,\{V, Y\}_{S}\right\}_{S}$.

Theorem 7.8. Let $U$ be a topological space. The functor $\{U,-\}_{S}$ has a left adjoint which will be denoted by $U \otimes-$. Moreover:

1. one has the natural isomorphism of flows

$$
U \otimes\left(\underset{\vec{i}}{\lim _{i}} X_{i}\right) \cong \underset{i}{\lim }\left(U \otimes X_{i}\right)
$$

2. there is a natural isomorphism of flows $\{*\} \otimes Y \cong Y$

3. if $Z$ is a topological space, one has the natural isomorphism of flows

$$
U \otimes \operatorname{Glob}(Z) \cong \operatorname{Glob}(U \times Z)
$$

4. for any flow $X$ and any topological space $U$, one has the natural bijection of sets

$$
(U \otimes X)^{0} \cong X^{0}
$$

5. if $U$ and $V$ are two topological spaces, then $(U \times V) \otimes Y \cong U \otimes(V \otimes Y)$ as flows

6. for any flow $X, \varnothing \otimes X \cong X^{0}$.

If $u \in U$, the image of $x \in X$ by the canonical morphism of flows $X \longrightarrow$ $\{u\} \otimes X \longrightarrow U \otimes X$ is denoted by $u \otimes x$.

Proof. In the category of Flow, let us start with the class of solutions $f: Z \longrightarrow$ $\{U, Y\}_{S}$ for $f$ running over the set Flow $\left(Z,\{U, Y\}_{S}\right)$ and for $Y$ running over the class of flows. Consider the commutative diagram

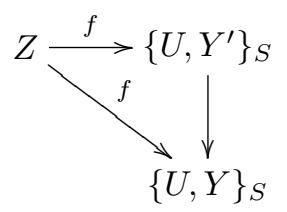

where $Y^{\prime}$ is the subflow generated by the elements of $f(Z)(U)$ and where the vertical map is induced by the inclusion $Y^{\prime} \subset Y$. So one still has a set of solutions by considering only the flows $Y$ such that the cardinal $\operatorname{card}(Y)$ of the underlying set satisfies $\operatorname{card}(Y) \leqslant \aleph_{0} \times \operatorname{card}(Z) \times \operatorname{card}(U)$. Let $\left\{Z_{i}, i \in I\right\}$ be the set of isomorphism classes of flows whose underlying set is of cardinal less than $\aleph_{0} \times \operatorname{card}(Z) \times \operatorname{card}(U)$. Then $\operatorname{card}(I) \leqslant 2^{\left(\aleph_{0} \times \operatorname{card}(Z) \times \operatorname{card}(U)\right)^{5}}$ so $I$ is a set. Then the class $\bigcup_{i \in I}$ Flow $\left(Z,\left\{U, Z_{i}\right\}_{S}\right)$ is a set as well and one gets a set of solutions. The first assertion is then clear using 
Theorem 4.16. One has Flow $(\{*\} \otimes X, Y) \cong$ Flow $\left(X,\{\{*\}, Y\}_{S}\right) \cong$ Flow $(X, Y)$ so by Yoneda $\{*\} \otimes X \cong X$ for any flow $X$. One has

$$
\text { Flow } \begin{aligned}
(U \otimes \operatorname{Glob}(Z), Y) & \cong \text { Flow }\left(\operatorname{Glob}(Z),\{U, Y\}_{S}\right) \\
& \cong \bigsqcup_{(\alpha, \beta) \in Y^{0} \times Y^{0}} \operatorname{Top}\left(Z, \mathbf{T O P}\left(U, \mathbb{P}_{\alpha, \beta} Y\right)\right) \\
& \cong \bigsqcup_{(\alpha, \beta) \in Y^{0} \times Y^{0}} \operatorname{Top}\left(U \times Z, \mathbb{P}_{\alpha, \beta} Y\right) \\
& \cong \text { Flow }(\operatorname{Glob}(U \times Z), Y)
\end{aligned}
$$

So by Yoneda $U \otimes \operatorname{Glob}(Z) \cong \operatorname{Glob}(U \times Z)$. One has

$$
\text { Flow } \begin{aligned}
(U \otimes\{*\}, Y) & \cong \text { Flow }\left(\{*\},\{\{*\}, Y\}_{S}\right) \\
& \cong\{\{*\}, Y\}_{S}^{0} \\
& \cong \text { Flow }(\{*\}, Y)
\end{aligned}
$$

so by Yoneda, $U \otimes\{*\} \cong\{*\}$. Hence $(U \otimes X)^{0} \cong X^{0}$ if $X$ is a point or a globe. Hence the result by Corollary 6.2. One has

$$
\text { Flow } \begin{aligned}
((U \times V) \otimes X, Y) & \cong \text { Flow }\left(X,\{V \times U, Y\}_{S}\right) \\
& \cong \text { Flow }\left(X,\left\{V,\{U, Y\}_{S}\right\}_{S}\right) \\
& \cong \text { Flow }\left(V \otimes X,\{U, Y\}_{S}\right) \\
& \cong \text { Flow }(U \otimes(V \otimes X), Y)
\end{aligned}
$$

so by Yoneda $(U \times V) \otimes X \cong U \otimes(V \otimes X)$.

Take a flow $X$ and a topological space $U$. One knows that $X$ is the colimit in a canonical way of points and globes (Theorem 6.1). Since $U \otimes\{*\} \cong\{*\}$ and $U \otimes \operatorname{Glob}(Z) \cong \operatorname{Glob}(U \times Z)$, and since the functor $U \otimes-$ commutes with colimits, one can represent $U \otimes X$ as the colimit of the diagram of Figure 6 with an obvious definition of the arrows (in particular $r_{U}^{\alpha, \beta, \gamma}$ uses the diagonal $U \longrightarrow U \times U$ ).

\subsection{Cylinder functor for the S-homotopy of flows}

Theorem 7.9. Let $U$ be a connected non-empty topological space. Let $X$ and $Y$ be two flows. Then one has a natural bijection of sets

$$
\text { Flow }\left(X,\{U, Y\}_{S}\right) \cong \operatorname{Top}(U, \mathbf{F L O W}(X, Y))
$$

and so

$$
\text { Flow }(U \otimes X, Y) \cong \operatorname{Top}(U, \mathbf{F L O W}(X, Y)) .
$$

Proof. It suffices to prove the first bijection by Theorem 7.8. Let

$$
f \in \text { Flow }\left(X,\{U, Y\}_{S}\right) .
$$

Then $f$ induces a set map from $X^{0}$ to $Y^{0}$ (but $Y^{0} \cong \operatorname{Top}\left(U, Y^{0}\right)$ since $U$ is connected) and a continuous map from $\mathbb{P} X$ to $\mathbf{T O P}(U, \mathbb{P} Y)$. So one has the inclusion 


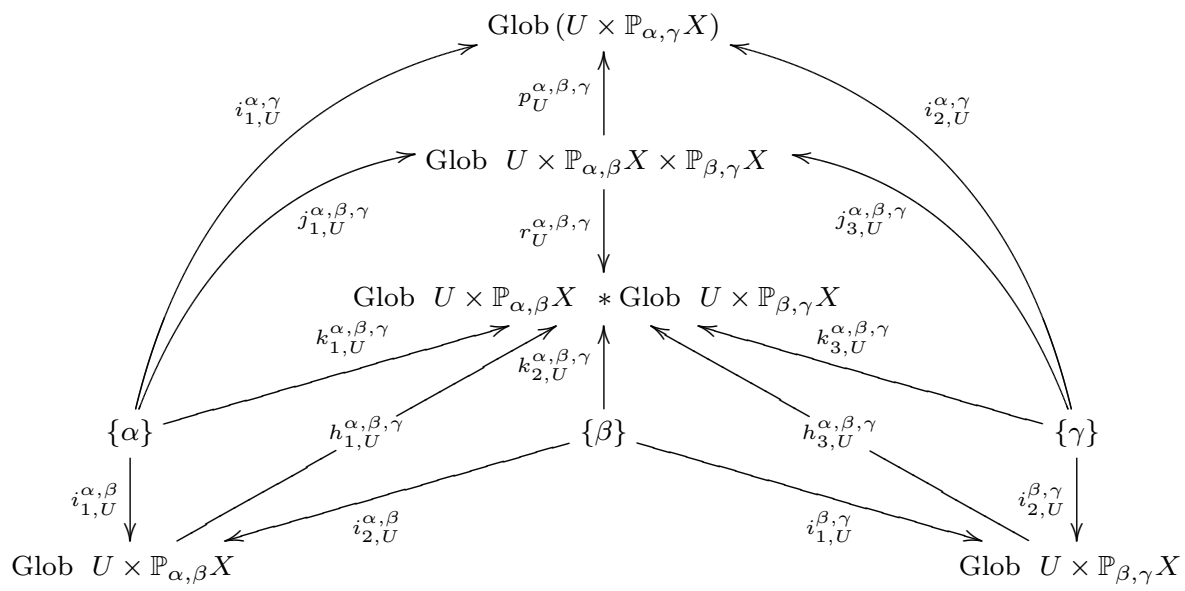

Figure 6: Representation of the flow $U \otimes X$

of sets

$$
i_{1}: \text { Flow }\left(X,\{U, Y\}_{S}\right) \subset \operatorname{Top}(X, \mathbf{T O P}(U, Y)) .
$$

The inclusion of sets FLOW $(X, Y) \longrightarrow$ TOP $(X, Y)$ induces an inclusion of sets

$$
i_{2}: \operatorname{Top}(U, \mathbf{F L O W}(X, Y)) \longrightarrow \operatorname{Top}(U, \mathbf{T O P}(X, Y)) .
$$

$\operatorname{But} \mathbf{T o p}(X, \mathbf{T O P}(U, Y)) \cong \operatorname{Top}(U, \mathbf{T O P}(X, Y))$. And it is then easy to see that $i_{1}$ and $i_{2}$ have the same image. So the sets Flow $\left(X,\{U, Y\}_{S}\right)$ and

\section{Top $(U$, FLOW $(X, Y))$}

are bijective.

Definition 7.10. Let $\mathcal{C}$ be a category. A cylinder is a functor $I: \mathcal{C} \longrightarrow \mathcal{C}$ together with natural transformations $i_{0}, i_{1}: \operatorname{Id}_{\mathcal{C}} \longrightarrow I$ and $p: I \longrightarrow \operatorname{Id}_{\mathcal{C}}$ such that $p \circ i_{0}$ and $p \circ i_{1}$ are the identity natural transformation.

Corollary 7.11 (Cylinder functor). The mapping $X \mapsto[0,1] \bigotimes X$ induces a functor from Flow to itself which is a cylinder functor with the natural transformations $e_{i}:\{i\} \otimes-\longrightarrow[0,1] \otimes-$ induced by the inclusion maps $\{i\} \subset[0,1]$ for $i \in\{0,1\}$ and with the natural transformation $p:[0,1] \otimes-\longrightarrow\{0\} \otimes-$ induced by the constant map $[0,1] \longrightarrow\{0\}$. Moreover, two morphisms of flows $f$ and $g$ from $X$ to $Y$ are S-homotopic if and only if there exists a morphism of flows $H:[0,1] \otimes X \longrightarrow Y$ such that $H \circ e_{0}=f$ and $H \circ e_{1}=g$. Moreover $e_{0} \circ H \sim_{S}$ Id and $e_{1} \circ H \sim_{S}$ Id.

Proof. Consequence of Theorem 7.9, Proposition 7.5 and Theorem 7.8 and of the connectedness of $[0,1]$. 


\section{Explicit description of $U \otimes X$}

Proposition 8.1. Let $X$ and $Y$ be two flows. Let $U$ be a topological space. Then one has a bijection between the elements of Flow $(U \otimes X, Y)$ and the elements $f$ of Set $\left(X^{0}, Y^{0}\right) \times \operatorname{Top}(U \times \mathbb{P} X, Y)$ such that

- $f\left(X^{0}\right) \subset Y^{0}$

- $f(U \times \mathbb{P} X) \subset \mathbb{P} Y$

- for any $u \in U, f(u, x * y)=f(u, x) * f(u, y)$ if $x, y \in \mathbb{P} X$ and if $t x=s y$

- for any $u \in U, s(f(u, x))=f(s(x))$ and $t(f(u, x))=f(t(x))$ if $x \in \mathbb{P} X$.

Proof. The set Flow $(U \otimes X, Y)$ is isomorphic to the set Flow $\left(X,\{U, Y\}_{S}\right)$, hence the result.

Proposition 8.2. The forgetful functor $v$ from Flow to the category of diagrams $D$ of topological spaces over the small category $1 \stackrel{s}{\longrightarrow} 0<^{t}-1$ such that $D(0)$ is a discrete topological space has a left adjoint called the free flow generated by the diagram.

Proof. The forgetful functor preserves limits because of the construction of the limit in Flow. Let $D$ be a diagram over $1 \stackrel{s}{\longrightarrow} 0 \stackrel{t}{\longleftarrow} 1$ with $D(0)$ discrete. Let us start from the class of solutions $f: D \longrightarrow v(X)$ when $X$ runs over the class of flows and for a given $X$ where $f$ runs over Flow $(D, v(X))$. Then one can replace $X$ by the subflow generated by the finite composition of elements of $f(D)$. So one can suppose that the cardinal $\operatorname{card}(X)$ of $X$ satisfies $\operatorname{card}(X) \leqslant \aleph_{0} \times \operatorname{card}(D)$ where $\operatorname{card}(D)$ is the cardinal of $D$. By choosing one equivalence class of flows for the class of flows $X$ such that $\operatorname{card}(X) \leqslant \aleph_{0} \times \operatorname{card}(D)$, one has obtained a set of solutions. Hence the result by Theorem 4.16.

Corollary 8.3. Let $U$ be a topological space. Let $X$ be a flow. Then the flow $U \otimes X$ is the free flow generated by the diagram $D$ of spaces defined by $D(1)=U \times \mathbb{P} X$, $D(0)=X^{0}, s(u, x)=s(x), t(u, x)=t(x)$ divided by the identifications $(u, x) *$ $(u, y)=(u, x * y)$.

Proof. The identifications generates an equivalence with closed graph since the composition law of $X$ is continuous. Therefore the quotient equipped with the final topology is still weak Hausdorff, and therefore compactly generated. This is then a consequence of Yoneda's lemma.

\section{S-homotopy extension property}

Definition 9.1. Let $i: A \longrightarrow X$ be a synchronized morphism of flows and let $Y$ be a flow. The morphism $i: A \longrightarrow X$ satisfies the S-homotopy extension property for $Y$ if for any morphism $f: X \longrightarrow Y$ and any S-homotopy $h:[0,1] \otimes A \longrightarrow Y$ such that for any $a \in A, h(0 \otimes a)=f(i(a))$, there exists a S-homotopy $H:[0,1] \otimes X \longrightarrow$ $Y$ such that for any $x \in X, H(0 \otimes x)=f(x)$ and for any $(t, a) \in[0,1] \times A$, $H(t \otimes i(a))=h(t \otimes a)$. 
Definition 9.2. A synchronized morphism $i: A \longrightarrow X$ satisfies the S-homotopy extension property if $i: A \longrightarrow X$ satisfies the $S$-homotopy extension property for any flow $Y$.

Let $i: A \longrightarrow X$ be a morphism of flows and let $(Y, B)$ be a pair of topological spaces. Then one can consider the pushout

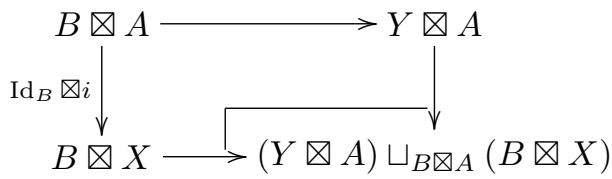

The commutativity of the diagram

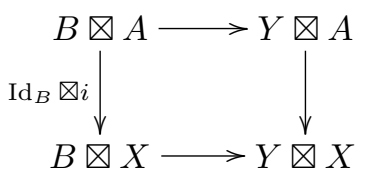

provides a canonical morphism of flows $(Y, B) \otimes i:(Y \otimes A) \sqcup_{B \otimes A}(B \otimes X) \longrightarrow Y \otimes X$ such that

$$
\begin{aligned}
& ((Y, B) \otimes i)(y \otimes a)=y \otimes i(a), \\
& ((Y, B) \otimes i)(b \otimes a)=b \otimes i(a), \\
& ((Y, B) \otimes i)(b \otimes x)=b \otimes x
\end{aligned}
$$

with $x \in X, y \in Y, a \in A$ and $b \in B$.

Notation 9.3. The morphism of flows $([0,1],\{0\}) \otimes i$ will be denoted by $\psi(i)$.

Theorem 9.4. Let $i: A \longrightarrow X$ be a morphism of flows. Then the following assertions are equivalent:

1. the morphism $i$ satisfies the S-homotopy extension property

2. the morphism of flows $\psi(i)$ has a retract $r$, that is to say there exists a morphism of flows

$$
r:[0,1] \otimes X \longrightarrow([0,1] \otimes A) \sqcup_{\{0\} \otimes A}(\{0\} \otimes X)
$$

such that $r \circ \psi(i)=\operatorname{Id}_{([0,1] \otimes A)} \sqcup_{\{0\} \otimes A}(\{0\} \otimes X)$.

Proof. Giving two morphisms of flows $f: X \longrightarrow Y$ and $h:[0,1] \otimes A \longrightarrow Y$ such that $h(0 \otimes a)=f(i(a))$ for any $a \in A$ is equivalent to giving a morphism of flows still denoted by $h$ from $([0,1] \otimes A) \sqcup_{\{0\} \otimes A}(\{0\} \otimes X)$ to $Y$. The S-homotopy extension problem for $i$ has then always a solution if and only for any morphism of flows $h:([0,1] \otimes A) \sqcup_{\{0\} \otimes A}(\{0\} \otimes X) \longrightarrow Y$, there exists a morphism of flows $H:[0,1] \otimes X \longrightarrow Y$ such that $H \circ \psi(i)=h$. Take $Y=([0,1] \otimes A) \sqcup_{\{0\} \otimes A}(\{0\} \otimes X)$ and let $h$ be the identity map of $Y$. This yields the retract $r$. Conversely, let $r$ be a retract of $i$. Then $H:=h \circ r$ is always a solution of the S-homotopy extension problem. 
Theorem 9.5. Let $(Z, \partial Z)$ be a NDR pair of topological spaces. Then the canonical morphism of flows $\operatorname{Glob}(\partial Z) \longrightarrow \operatorname{Glob}(Z)$ satisfies the S-homotopy extension property.

Proof. Since $(Z, \partial Z)$ is a NDR pair, then $[0,1] \times \partial Z \sqcup_{\{0\} \times \partial Z} Z \longrightarrow[0,1] \times Z$ has a retract. Therefore the morphism of flows

$$
\operatorname{Glob}\left([0,1] \times \partial Z \sqcup_{\{0\} \times \partial Z} Z\right) \longrightarrow \operatorname{Glob}([0,1] \times Z)
$$

has a retract. But

$$
\operatorname{Glob}\left([0,1] \times \partial Z \sqcup_{\{0\} \times \partial Z} Z\right) \cong[0,1] \otimes \operatorname{Glob}(\partial Z) \sqcup_{\{0\} \otimes \operatorname{Glob}(\partial Z)} \operatorname{Glob}(Z)
$$

and Glob $([0,1] \times Z) \cong[0,1] \otimes \operatorname{Glob}(Z)$. The proof is complete thanks to Theorem 9.4.

Theorem 9.6. Let $U$ be a connected non empty space. Let $X$ and $Y$ be two flows. Then there exists a natural homeomorphism

$$
\operatorname{TOP}(U, \mathbf{F L O W}(X, Y)) \cong \mathbf{F L O W}(U \otimes X, Y) .
$$

Proof. We already know by Theorem 7.9 that there exists a natural bijection

$$
\operatorname{Top}(U, \mathbf{F L O W}(X, Y)) \cong \operatorname{glTop}(U \otimes X, Y) .
$$

Using the construction of $\otimes$, Corollary 6.2 and Theorem 5.10, it suffices to prove the homeomorphism for $X=X^{0}$ and $X=\operatorname{Glob}(Z)$. The space $\mathbf{F L O W}\left(X^{0}, Y\right)$ is the discrete space of set maps $\operatorname{Set}\left(X^{0}, Y^{0}\right)$ from $X^{0}$ to $Y^{0}$. Since $U$ is connected, then $\operatorname{TOP}\left(U, \mathbf{F L O W}\left(X^{0}, Y\right)\right) \cong \operatorname{Set}\left(X^{0}, Y^{0}\right)$. In the other hand, FLOW $\left(U \otimes X^{0}, Y\right) \cong$ $\mathbf{F L O W}\left(X^{0}, Y\right) \cong \operatorname{Set}\left(X^{0}, Y^{0}\right)$, hence the result for $X^{0}$. At last, for any topological space $W$,

$$
\begin{aligned}
& \operatorname{Top}(W, \text { TOP }(U, \text { FLOW }(\operatorname{Glob}(Z), Y))) \\
& \cong \text { Top }(W \times U, \text { FLOW }(\operatorname{Glob}(Z), Y)) \\
& \cong \operatorname{glTop}((W \times U) \otimes \operatorname{Glob}(Z), Y) \\
& \cong \operatorname{glTop}(\operatorname{Glob}(W \times U \times Z), Y)
\end{aligned}
$$

and $\operatorname{Top}(W, \mathbf{F L O W}(U \otimes \operatorname{Glob}(Z), Y) \cong \operatorname{Top}(W, \mathbf{F L O W}(\operatorname{Glob}(U \times Z), Y))$. It is then easy to see that both

$$
\operatorname{glTop}(\operatorname{Glob}(W \times U \times Z), Y)
$$

and

$$
\operatorname{Top}(W, \mathbf{F L O W}(\operatorname{Glob}(U \times Z), Y))
$$

can be identified to the same subset of $\operatorname{Top}([0,1] \times W \times U \times Z, Y)$. Hence the result by Yoneda.

Theorem 9.7. A morphism of flows $i: A \longrightarrow X$ satisfies the $S$-homotopy extension property if and only if for any flow $Y$, the continuous map $i^{*}: \mathbf{F L O W}(X, Y) \longrightarrow$ FLOW $(A, Y)$ is a Hurewicz fibration. 
Proof. For any topological space $M$, one has

$$
\operatorname{Top}([0,1] \times M, \mathbf{F L O W}(A, Y)) \cong \operatorname{Top}(M, \boldsymbol{T O P}([0,1], \mathbf{F L O W}(A, Y)))
$$

since Top is cartesian closed and

$$
\operatorname{Top}(M, \mathbf{T O P}([0,1], \mathbf{F L O W}(A, Y))) \cong \operatorname{Top}(M, \mathbf{F L O W}([0,1] \otimes A, Y))
$$

by Theorem 9.6. Considering a commutative diagram like

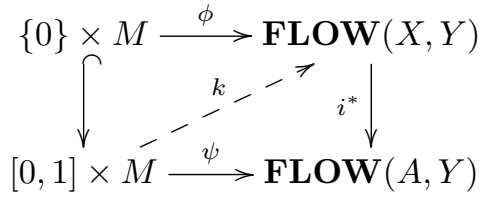

is then equivalent to considering a commutative diagram of topological spaces

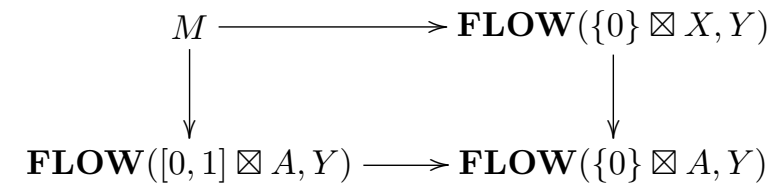

Using again Theorem 5.10, considering such a commutative diagram is equivalent to considering a continuous map $M \longrightarrow \mathbf{F L O W}(M i, Y)$. Finding a continuous map $k$ making both triangles commutative is equivalent to finding a commutative diagram of the form

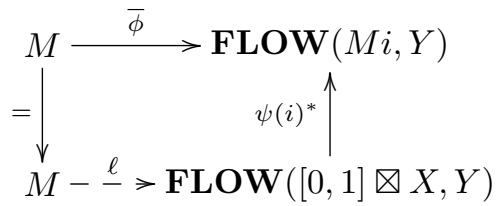

If $i: A \longrightarrow X$ satisfies the S-homotopy extension property, then $\psi(i): M i \longrightarrow$ $[0,1] \otimes X$ has a retract $r:[0,1] \otimes X \longrightarrow M i$. Then take $\ell=\bar{\phi} \circ r$. Conversely, if $\ell$ exists for any $M$ and any $Y$, take $M=\{0\}$ and $Y=M i$ and $\bar{\phi}(0)=\operatorname{Id}_{M i}$. Then $\ell(0)$ is a retract of $\psi(i)$. Therefore $i: A \longrightarrow X$ satisfies the S-homotopy extension property.

Corollary 9.8. Let $i: A \longrightarrow X$ satisfy the S-homotopy extension property. Let $f: A \longrightarrow Y$ be a morphism of flows. Consider the pushout in Flow

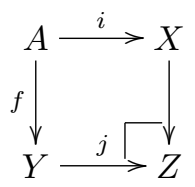

Then the canonical morphism from $Y$ to $Z$ satisfies the $S$-homotopy extension property. In other terms, the pushout of a morphism of flows satisfying the S-homotopy extension property still satisfies the S-homotopy extension property. 
Corollary 9.9. Let $Z$ be a compact space and let $\partial Z \subset Z$ be a compact subspace such that the canonical inclusion is a NDR pair. Let $U$ be a flow. Then the canonical restriction map

$$
\text { FLOW }(\operatorname{Glob}(Z), U) \longrightarrow \text { FLOW }(\operatorname{Glob}(\partial Z), U)
$$

is a Hurewicz fibration.

\section{Morphisms of flows inducing a closed inclusion of path spaces}

Proposition 10.1. [28] If $j: X \longrightarrow Y$ and $r: Y \longrightarrow X$ are two continuous maps with $r \circ j=\mathrm{Id}$, then $j$ is a closed inclusion and $r$ is a quotient map.

Notation 10.2. Denote by $\mathcal{I N C}$ the class of morphisms of flows $f: X \longrightarrow Y$ such that $\mathbb{P} f: \mathbb{P} X \longrightarrow \mathbb{P} Y$ is a closed inclusion of topological spaces.

The purpose of this section is to collect some important examples of morphisms of $\mathcal{I N C}$. This section provides the necessary preparatory lemmas for the use of the "Small Object Argument" further in this paper.

Proposition 10.3. Let $A$ be a flow. Then the morphism of flows $\theta: A \longrightarrow[0,1] \bowtie A$ defined by $\theta(a)=1 \otimes$ a belongs to $\mathcal{I N C}$.

Proof. The mapping $U \mapsto U \otimes X$ for a given flow $X$ is functorial with respect to $U$. So one can consider $r:[0,1] \otimes A \longrightarrow A$ defined by $r(t \otimes a)=a$.

Definition 10.4. If $i: A \longrightarrow X$ is a morphism of flows, then the mapping cylinder $M i$ of $i$ is defined by the pushout of flows

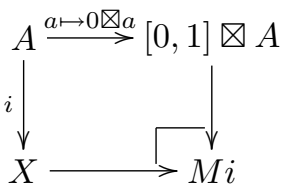

Proposition 10.5. Let $i: A \longrightarrow X$ be a synchronized morphism of flows. Then the canonical morphism of flows $\theta: A \longrightarrow$ Mi such that $\theta(a)=1 \otimes$ a belongs to $\mathcal{I N C}$.

Proof. First of all, since $i$ is synchronized, one can consider that $A^{0}=X^{0}$. Let

$$
N i=([0,1] \otimes A) \sqcup_{A^{0}} X .
$$

Then there exists a canonical morphism of flows $\phi: N i \longrightarrow M i$ which is constant on the 0-skeleton and such that $\phi: \mathbb{P} N i \longrightarrow \mathbb{P} M i$ is onto. Let us consider the equivalence relation $\mathcal{R}$ on $\mathbb{P} N i$ associated to $\phi$, i.e. $x \mathcal{R} y$ if and only if $\phi(x)=\phi(y)$. The graph of $\mathcal{R}$ is the inverse image of the diagonal of $\mathbb{P} M i$. The latter is closed in $\mathbb{P} M i \times \mathbb{P} M i$ since $\mathbb{P} M i$ is a $k$-space which is weak Hausdorff. Therefore the graph of $\mathcal{R}$ is closed in $\mathbb{P} N i \times \mathbb{P} N i$. So the quotient $\mathbb{P} N i / \mathcal{R}$ equipped with the final topology is still weak Hausdorff and thus a compactly generated topological space. There exists a canonical continuous map $\mathbb{P} N i / \mathcal{R} \longrightarrow \mathbb{P} M i$ which is an isomorphism of 
sets. The topological space $\mathbb{P} N i / \mathcal{R}$ yields a flow $Y$ and a commutative diagram of flows

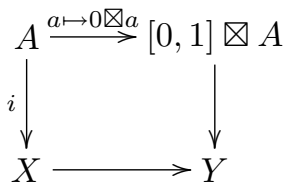

Therefore there exists a morphism of flows $M i \longrightarrow Y$ because of the universal property satisfied by $M i$. So the bijection $\sigma: \mathbb{P} N i / \mathcal{R} \longrightarrow \mathbb{P} M i$ is actually an homeomorphism and the continuous map $\phi: \mathbb{P} N i \longrightarrow \mathbb{P} M i$ is a quotient map. The $\operatorname{map} \theta: A \longrightarrow M i$ is equal to the composite

$$
A \cong\{1\} \otimes A \longrightarrow N i \longrightarrow M i
$$

One has $\mathbb{P} N i \cong \mathbb{P}([0,1] \otimes A) \sqcup Z$ for some topological space $Z$ : the topological space $Z$ consists of all free compositions of executions paths of $N i$ containing an element of $X$. Therefore the morphism of flows $A \longrightarrow N i$ is a closed inclusion of topological spaces. Let $g: Z \longrightarrow A$ be a set map such that $\theta \circ g: Z \longrightarrow \mathbb{P} M i$ is continuous. Let $F$ be a closed subspace of $A$. Then $F$ is mapped to a closed subspace $G$ of $\mathbb{P} N i$. Since $G=\sigma^{-1}(\sigma(G))$, then $\sigma(G)$ is a closed subspace of $\mathbb{P} M i$. Therefore $g^{-1}(F)=(\theta \circ g)^{-1}(\sigma(G))$ is a closed subspace of $Z$. Therefore $g$ is continuous.

Theorem 10.6. Let $i: A \longrightarrow X$ satisfy the S-homotopy extension property. Then $i \in \mathcal{I N C}$.

Proof. We follow the proof of the fact that any Hurewicz cofibration of compactly topological spaces is a closed inclusion given in the appendix of $[\mathbf{2 8}]$.

Let us consider the commutative diagram of flows

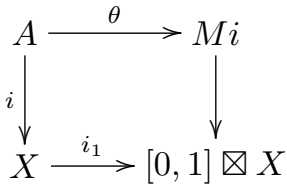

where $\theta(a)=1 \otimes a$ and $i_{1}(x)=1 \bigotimes x$. Then $i_{1}$ has a retract and therefore is a closed inclusion. The map $\theta$ is a closed inclusion as well by Proposition 10.5. Since $j$ has a retract by Theorem 9.4, then $j \circ \theta$ is a closed inclusion. moreover $i_{1}$ is one-to-one. Therefore $i$ is a closed inclusion.

\section{Smallness argument}

Any ordinal can be viewed as a small category whose objects are the elements of $\lambda$, that is the ordinal $\gamma<\lambda$, and where there exists a morphism $\gamma \longrightarrow \gamma^{\prime}$ if and only if $\gamma \leqslant \gamma^{\prime}$.

Definition 11.1. Let $\mathcal{C}$ be a cocomplete category. Let $\lambda$ be an ordinal. $A \lambda$-sequence in $\mathcal{C}$ is a colimit-preserving functor $X: \lambda \longrightarrow \mathcal{C}$. Since $X$ preserves colimits, for all limit ordinals $\gamma<\lambda$, the induces map $\lim _{\beta<\gamma} X_{\beta} \longrightarrow X_{\gamma}$ is an isomorphism. The morphism $X_{0} \longrightarrow \lim _{\longrightarrow} X$ is called the transfinite composition of the $X_{\gamma} \longrightarrow X_{\gamma+1}$. 
Definition 11.2. Let $\kappa$ be a cardinal. An ordinal $\lambda$ is $\kappa$-filtered if for any $A \subset \lambda$ with $|A| \leqslant \kappa$ where $|A|$ is the cardinal of $A$, then $\sup A<\lambda$.

Definition 11.3. Let $\mathcal{C}$ be a cocomplete category. Let $\mathcal{D}$ be a collection of morphisms of $\mathcal{C}$. Let $\kappa$ be a cardinal. An object $A$ of $\mathcal{C}$ is $\kappa$-small with respect to $\mathcal{D}$ if for any $\lambda$ sequence $X$ where $\lambda$ is a $\kappa$-filtered ordinal, and where each arrow $X_{\beta} \longrightarrow X_{\beta+1}$ lies in $\mathcal{D}$ for $\beta<\lambda$, then one has the bijection $\lim _{\beta<\lambda} \mathcal{C}\left(A, X_{\beta}\right) \longrightarrow \mathcal{C}\left(A, \lim _{\beta<\lambda} X_{\beta}\right)$. $W$ e say that $A$ is $\kappa$-small relative to $\mathcal{D}$ if it is $\kappa$-small relative to $\mathcal{D}$ for some cardinal $\kappa$.

Definition 11.4. Let $\mathcal{C}$ be a cocomplete category. Let I be a set of morphisms of $\mathcal{C}$. Then a relative $I$-cell complex $f: A \longrightarrow B$ is a transfinite composition of pushouts of elements of $I$. In other terms, there exists an ordinal $\lambda$ and a $\lambda$-sequence $X: \lambda \longrightarrow \mathcal{C}$ such that $f$ is the composition of $X$ and such that for each $\beta$ with $\beta+1<\lambda$, there is a pushout square as follows

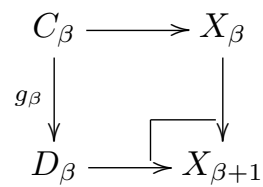

such that $g_{\beta} \in I$. We denote the collection of relative $I$-cell complexes by I-cell. If $\varnothing$ is the initial object of $\mathcal{C}$ and if $X$ is an object of $\mathcal{C}$ such that $\varnothing \longrightarrow X$ is a relative $I$-cell complex, then one says that $X$ is a I-cell complex.

Proposition 11.5. Any flow $A$ is $\sup \left(\aleph_{0}, \operatorname{card}(A)\right)$-small relative to $\mathcal{I N C}$ where $\operatorname{card}(A)$ is the cardinal of the underlying topological space of $A$.

Proof. One has a canonical one-to-one set map

$$
\underset{\beta<\lambda}{\lim _{\beta<\lambda}} \operatorname{Flow}\left(A, X_{\beta}\right) \longrightarrow \operatorname{Flow}\left(A, \underset{\beta<\lambda}{\lim _{\beta}} X_{\beta}\right) .
$$

Let $f \in \operatorname{Flow}\left(A, \lim _{\beta<\lambda} X_{\beta}\right)$. Since the 0 -skeleton of a colimit of flows is the colimit of the 0-skeletons, then for any $a \in A^{0}, f(a) \in X_{\beta_{a}}^{0}$ for some $\beta_{a}<\lambda$. There exists a canonical continuous map $\lim _{\beta<\lambda} \mathbb{P} X_{\beta} \longrightarrow \mathbb{P}\left(\underline{\lim }_{\beta<\lambda} X_{\beta}\right)$ where $\lim _{\beta<\lambda} X_{\beta}$ is the colimit of the flows $X_{\beta}$. Any element of $\mathbb{P}\left(\lim _{X<\lambda} X_{\beta}\right)$ is a finite composite $x_{1} * \cdots * x_{r}$ of elements $x_{1} \in X_{\beta_{1}}, \ldots, x_{r} \in X_{\beta_{r}}$ for some finite integer $r$. Since $\lambda$ is $\sup \left(\aleph_{0}, \operatorname{card}(A)\right)$-filtered, it is $\aleph_{0}$-filtered. So $\beta=\sup \left(\beta_{1}, \ldots, \beta_{r}\right)<\lambda$ and $x_{1}, \ldots, x_{r} \in \mathbb{P} X_{\beta}$. So $x_{1} * \cdots * x_{r} \in \mathbb{P} X_{\beta}$. Therefore any execution path $x \in$ $\mathbb{P}\left(\mathrm{lim}_{\beta<\lambda} X_{\beta}\right)$ belongs to some $\mathbb{P} X_{\beta_{x}}$ for some $\beta_{x}<\lambda$. Since $\lambda$ is $\sup \left(\aleph_{0}, \operatorname{card}(A)\right)$ filtered, it is $\operatorname{card}(A)$-filtered. Therefore $\sup \left(\beta_{a}, \ldots, \beta_{x}\right)<\lambda$. So $f$ factors through a map $g: A \longrightarrow X_{\beta}$ with $\beta<\lambda$. The map $g: A \longrightarrow X_{\beta}$ is automatically continuous because all continuous maps between path spaces are inclusions of topological spaces.

Definition 11.6. A morphism of flows $f: X \longrightarrow Y$ is a weak S-homotopy equivalence if $f$ is synchronized and if $f$ induces a weak homotopy equivalence from $\mathbb{P} X$ to $\mathbb{P} Y$. 
Notation 11.7. Let $\mathcal{S}$ be the subcategory of weak S-homotopy equivalences. Let $I^{g l}$ be the set of morphisms of flows $\operatorname{Glob}\left(\mathbf{S}^{n-1}\right) \longrightarrow \operatorname{Glob}\left(\mathbf{D}^{n}\right)$ for $n \geqslant 0$. Let $J^{\text {gl }}$ be the set of morphisms of flows $\operatorname{Glob}\left(\mathbf{D}^{n}\right) \longrightarrow \operatorname{Glob}\left([0,1] \times \mathbf{D}^{n}\right)$. Notice that all arrows of $\mathcal{S}, I^{g l}$ and $J^{g l}$ are synchronized. At last, denote by $I_{+}^{g l}$ be the union of $I^{g l}$ with the two morphisms of flows $R:\{0,1\} \longrightarrow\{0\}$ and $C: \varnothing \subset\{0\}$.

Proposition 11.8. The domains of $I_{+}^{g l}$ are small relative to $I_{+}^{g l}$-cell. The domains of $J^{g l}$ are small relative to $J^{g l}$-cell.

Proof. The inclusion maps $\mathbf{S}^{n-1} \subset \mathbf{D}^{n}$ and $\mathbf{D}^{n} \subset[0,1] \times \mathbf{D}^{n}$ are NDR pairs. So any pushout of a morphism of $I^{g l} \cup J^{g l}$ satisfies the S-homotopy extension property by Corollary 9.8 and Theorem 9.5, and therefore is an element of $\mathcal{I N C}$ by Theorem 10.6. A pushout of $C: \varnothing \longrightarrow\{0\}$ does not change the path space. Therefore such a pushout is necessarily in $\mathcal{I N C}$. It remains to examine the case of a pushout of $R:\{0,1\} \longrightarrow\{0\}$. Let us consider the pushout of flows

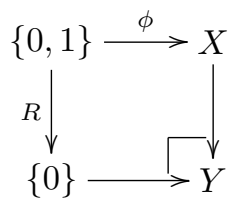

If $\phi(0)=\phi(1)$, then $\mathbb{P} X=\mathbb{P} Y$ and so there is nothing to prove. Otherwise, if $\phi(0) \neq$ $\phi(1)$, then $\mathbb{P} Y \cong \mathbb{P} X \sqcup\left(\mathbb{P}_{., \phi(1)} X \times \mathbb{P}_{\phi(0), .} X\right) \sqcup\left(\mathbb{P}_{., \phi(1)} X \times \mathbb{P}_{\phi(0), \phi(1)} X \times \mathbb{P}_{\phi(0), .} X\right) \sqcup \ldots$ Hence the conclusion by Proposition 11.5.

\section{Reminder about model category}

Some useful references for the notion of model category are $[\mathbf{2 7}][\mathbf{2 1}]$. See also [9] [26].

If $\mathcal{C}$ is a category, one denotes by $\operatorname{Map}(\mathcal{C})$ the category whose objects are the morphisms of $\mathcal{C}$ and whose morphisms are the commutative squares of $\mathcal{C}$.

In a category $\mathcal{C}$, an object $x$ is a retract of an object $y$ if there exists $f: x \longrightarrow y$ and $g: y \longrightarrow x$ of $\mathcal{C}$ such that $g \circ f=\operatorname{Id}_{x}$. A functorial factorization $(\alpha, \beta)$ of $\mathcal{C}$ is a pair of functors from $\operatorname{Map}(\mathcal{C})$ to $\operatorname{Map}(\mathcal{C})$ such that for any $f$ object of $\operatorname{Map}(\mathcal{C})$, $f=\beta(f) \circ \alpha(f)$.

Definition 12.1. Let $i: A \longrightarrow B$ and $p: X \longrightarrow Y$ be maps in a category $\mathcal{C}$. Then $i$ has the left lifting property (LLP) with respect to $p$ (or $p$ has the right lifting property (RLP) with respect to $i$ ) if for any commutative square

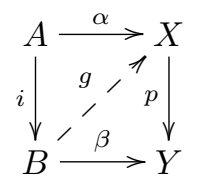

there exists $g$ making both triangles commutative. 
Definition 12.2. If I is a set of morphisms of flows, the collection of morphisms of flows that satisfies the RLP with respect to any morphism of I is denoted by I-inj. Denote by I-cof the collection of morphisms of flows that satisfies the RLP with respect to any morphism that satisfies the LLP with respect to any element of $I$. This is a purely categorical fact that $I-$ cell $\subset I-\operatorname{cof}$.

Definition 12.3. A model structure on a category $\mathcal{C}$ consists of three subcategories of the category of morphisms $\operatorname{Map}(\mathcal{C})$ called weak equivalences, cofibrations, and fibrations, and two functorial factorizations $(\alpha, \beta)$ and $(\gamma, \delta)$ satisfying the following properties:

1. (2-out-of-3) If $f$ and $g$ are morphisms of $\mathcal{C}$ such that $g \circ f$ is defined and two of $f, g$ and $g \circ f$ are weak equivalences, then so is the third.

2. (Retracts) If $f$ and $g$ are morphisms of $\mathcal{C}$ such that $f$ is a retract of $g$ and $g$ is a weak equivalence, cofibration, or fibration, then so is $f$.

3. (Lifting) Define a map to be a trivial cofibration if it is both a cofibration and a weak equivalence. Similarly, define a map to be a trivial fibration if it is both a fibration and a weak equivalence. Then trivial cofibrations have the LLP with respect to fibrations, and cofibrations have the LLP with respect to trivial fibrations.

4. (Factorization) For any morphism $f, \alpha(f)$ is a cofibration, $\beta(f)$ a trivial fibration, $\gamma(f)$ is a trivial cofibration, and $\delta(f)$ is a fibration.

Definition 12.4. A model category is a complete and cocomplete category $\mathcal{C}$ together with a model structure on $\mathcal{C}$.

Theorem 12.5. [27] Let $\mathcal{C}$ be a complete and cocomplete category. Let $\mathcal{W}$ be a subcategory of $\operatorname{Map}(\mathcal{C})$. Let $I$ and $J$ be two sets of maps of $\mathcal{C}$. Then there exists a structure of model category on $\mathcal{C}$ such that the fibrations are exactly the arrows satisfying the RLP with respect to the arrows of $J$, such that the trivial fibrations are exactly the arrows satisfying the RLP with respect to the arrows of $I$, such that the weak equivalences are exactly the arrows of $\mathcal{W}$ if the following conditions are satisfied:

1. The subcategory $\mathcal{W}$ has the 2-out-of-3 property and is closed under retracts.

2. The domains of I are small relative to I-cell.

3. The domains of $J$ are small relative to $J$-cell.

4. Any relative $J$-cell complex is a weak equivalence and satisfies the LLP with respect to any morphism satisfying the RLP with respect to the arrows of $I$. In other terms, $J-$ cell $\subset I-\operatorname{cof} \cap \mathcal{W}$.

5. A morphism satisfies the RLP with respect to the morphisms of I if and only if it is a weak equivalence and it satisfies the RLP with respect to the morphisms of $J$. In other terms, $I-i n j=J-i n j \cap \mathcal{W}$.

Definition 12.6. If the conditions of Theorem 12.5 are satisfied for some model category $\mathcal{C}$, the set $I$ is the set of generating cofibrations, the set $J$ is the set of generating trivial cofibrations and one says that $\mathcal{C}$ is a cofibrantly generated model category. 
The above conditions are satisfied for Top if $\mathcal{W}$ is the subcategory of weak homotopy equivalences, if $I$ is the set of inclusion maps $\mathbf{S}^{n-1} \longrightarrow \mathbf{D}^{n}$ with $\mathbf{S}^{-1}=\varnothing$ and for $n \geqslant 0$, and if $J$ is the set of continuous maps $\mathbf{D}^{n} \longrightarrow[0,1] \times \mathbf{D}^{n}$ such that $x \mapsto(0, x)$ and for $n \geqslant 0$. The fibrations of the model structure of Top are usually called Serre fibration.

So far, we have proved:

Theorem 12.7. The category of flows Flow is complete and cocomplete. Moreover:

1. The subcategory $\mathcal{S}$ has the 2-out-of-3 property and is closed under retracts.

2. The domains of $I_{+}^{g l}$ are small relative to $I_{+}^{g l}$-cell.

3. The domains of $J^{g l}$ are small relative to $J^{g l}$-cell.

\section{Characterization of the fibrations of flows}

Definition 13.1. An element of $J^{g l}-i n j$ is called a fibration. A fibration is trivial if it is at the same time a weak S-homotopy equivalence.

Proposition 13.2. A morphism of flows $f: X \longrightarrow Y$ satisfies the $R L P$ with respect to $\operatorname{Glob}(U) \longrightarrow \operatorname{Glob}(V)$ if and only if for any $\alpha, \beta \in X^{0}, \mathbb{P}_{\alpha, \beta} X \longrightarrow \mathbb{P}_{f(\alpha), f(\beta)} Y$ satisfies the $R L P$ with respect to $U \longrightarrow V$.

Proof. Considering a commutative square of topological spaces

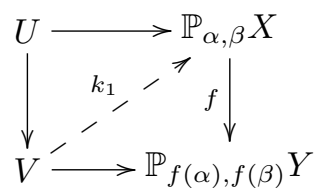

is equivalent to considering a commutative square of flows like

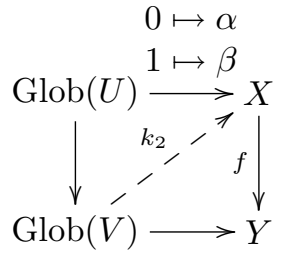

The existence of $k_{1}$ making the first diagram commutative is equivalent to the existence of $k_{2}$ making the second diagram commutative. Hence the result.

Proposition 13.3. Let $f: X \longrightarrow Y$ be a morphism of flows. Then $f$ is a fibration of flows if and only if $\mathbb{P} f: \mathbb{P} X \longrightarrow \mathbb{P Y}$ is a Serre fibration of topological spaces.

Proof. By Proposition 13.2, the morphism of flows $f$ is a fibration if and only if for any $\alpha, \beta \in X^{0}$, the continuous map $\mathbb{P}_{\alpha, \beta} X \longrightarrow \mathbb{P}_{f(\alpha), f(\beta)} Y$ is a Serre fibration. But $\mathbf{D}^{n}$ and $\mathbf{D}^{n} \times[0,1]$ are connected. Hence the result. 


\section{About the necessity of $R$ and $C$ as generating cofibrations}

One cannot take as definition of a cofibration an element of $I^{g l}-c o f$. Indeed:

Proposition 14.1. There does not exist any cofibrantly generated model structure on Flow such that the generating set of cofibrations is $I^{g l}$, the generating set of trivial cofibrations $\mathrm{J}^{g l}$, and the class of weak equivalences the one of weak S-homotopy equivalences.

Proof. If such a model structure existed, then all cofibrations would be synchronized because any cofibration is a retract of an element of $I^{g l}-c e l l$, because any element of $I^{g l}-c e l l$ is synchronized, and at last because the retract of a synchronized morphism of flows is synchronized. Since a trivial fibration is a weak S-homotopy equivalence, then such morphism is in particular synchronized. So all composites of the form $p \circ i$ where $p$ would be a trivial fibration and $i$ a cofibration would be synchronized. So a non-synchronized morphism of flows could never be equal to such composite.

Proposition 14.2. There does not exist any cofibrantly generated model structure on Flow such that the generating set of cofibrations is $I^{g l} \cup\{C\}$, the generating set of trivial cofibrations $J^{g l}$, and the class of weak equivalences the one of weak S-homotopy equivalences.

Proof. Suppose that such a model structure exists. Consider a commutative square

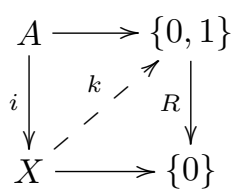

where $i: A \longrightarrow X$ is an element of $I^{g l} \cup\{C\}$. Since the path spaces of the flows $\{0,1\}$ and $\{0\}$ are empty, then $\mathbb{P} A=\mathbb{P} X=\varnothing$. So $i=C, A=\varnothing$ and $X=\{0\}$. Let $k(0)=0$. Then $k$ makes the diagram above commutative. Therefore $R$ satisfies the RLP with respect to any morphism of $I^{g l} \cup\{C\}$. So $R$ is a trivial fibration for this model structure. Contradiction.

Proposition 14.3. There does not exits any cofibrantly generated model structure on Flow such that the generating set of cofibrations is $I^{g l} \cup\{R\}$, the generating set of trivial cofibrations $J^{g l}$, and the class of weak equivalences the one of weak S-homotopy equivalences.

Proof. If such a model structure existed, then all cofibrations would restrict to an onto set map between the 0-skeletons. So there would not exist any cofibrant object since the initial flow is the empty set.

Hence the definition:

Definition 14.4. An element of $I_{+}^{g l}-$ cof is called a cofibration. A cofibration is trivial if it is at the same time a weak S-homotopy equivalence. 


\section{Pushout of $\operatorname{Glob}(\partial Z) \rightarrow \operatorname{Glob}(Z)$ in Flow}

Let $\partial Z \longrightarrow Z$ be a continuous map. Let us consider a diagram of flows as follows:

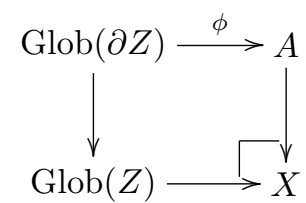

The purpose of this short section is an explicit description of the pushout $X$ in the category of flows.

Let us consider the set $\mathcal{M}$ of finite sequences $\alpha_{0} \ldots \alpha_{p}$ of elements of $A^{0}=$ $X^{0}$ with $p \geqslant 1$ and such that, for any $i$, at least one of the two pairs $\left(\alpha_{i}, \alpha_{i+1}\right)$ and $\left(\alpha_{i+1}, \alpha_{i+2}\right)$ is equal to $(\phi(0), \phi(1))$. Let us consider the pushout diagram of topological spaces

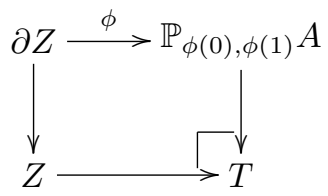

Let $Z_{\alpha, \beta}=\mathbb{P}_{\alpha, \beta} A$ if $(\alpha, \beta) \neq(\phi(0), \phi(1))$ and let $Z_{\phi(0), \phi(1)}=T$. At last, for any $\alpha_{0} \ldots \alpha_{p} \in \mathcal{M}$, let $\left[\alpha_{0} \ldots \alpha_{p}\right]=Z_{\alpha_{0}, \alpha_{1}} \times Z_{\alpha_{1}, \alpha_{2}} \times \ldots \times Z_{\alpha_{p-1}, \alpha_{p}}$. And $\left[\alpha_{0} \ldots \alpha_{p}\right]_{i}$ denotes the same product as $\left[\alpha_{0} \ldots \alpha_{p}\right]$ except that $\left(\alpha_{i}, \alpha_{i+1}\right)=(\phi(0), \phi(1))$ and that the factor $Z_{\alpha_{i}, \alpha_{i+1}}=T$ is replaced by $\mathbb{P}_{\phi(0), \phi(1)} A$. We mean that in the product $\left[\alpha_{0} \ldots \alpha_{p}\right]_{i}$, the factor $\mathbb{P}_{\phi(0), \phi(1)} A$ appears exactly once. For instance, one has (with $\phi(0) \neq \phi(1))$

$$
\begin{aligned}
& {[\alpha \phi(0) \phi(1) \phi(0) \phi(1)]=\mathbb{P}_{\alpha, \phi(0)} A \times T \times \mathbb{P}_{\phi(1), \phi(0)} A \times T} \\
& {[\alpha \phi(0) \phi(1) \phi(0) \phi(1)]_{1}=\mathbb{P}_{\alpha, \phi(0)} A \times \mathbb{P}_{\phi(0), \phi(1)} A \times \mathbb{P}_{\phi(1), \phi(0)} A \times T} \\
& {[\alpha \phi(0) \phi(1) \phi(0) \phi(1)]_{3}=\mathbb{P}_{\alpha, \phi(0)} A \times T \times \mathbb{P}_{\phi(1), \phi(0)} A \times \mathbb{P}_{\phi(0), \phi(1)} A .}
\end{aligned}
$$

The idea is that in the products $\left[\alpha_{0} \ldots \alpha_{p}\right]$, there are no possible simplifications using the composition law of $A$. On the contrary, exactly one simplification is possible using the composition law of $A$ in the products $\left[\alpha_{0} \ldots \alpha_{p}\right]_{i}$. For instance, with the examples above, there exist continuous maps

$$
[\alpha \phi(0) \phi(1) \phi(0) \phi(1)]_{1} \longrightarrow[\alpha \phi(0) \phi(1)]
$$

and

$$
[\alpha \phi(0) \phi(1) \phi(0) \phi(1)]_{3} \longrightarrow[\alpha \phi(0) \phi(1) \phi(1)]
$$

induced by the composition law of $A$ and there exist continuous maps

$$
[\alpha \phi(0) \phi(1) \phi(0) \phi(1)]_{1} \longrightarrow[\alpha \phi(0) \phi(1) \phi(0) \phi(1)]
$$

and

$$
[\alpha \phi(0) \phi(1) \phi(0) \phi(1)]_{3} \longrightarrow[\alpha \phi(0) \phi(1) \phi(0) \phi(1)]
$$

induced by the continuous map $\mathbb{P}_{\phi(0), \phi(1)} A \longrightarrow T$. 
Let $\mathbb{P}_{\alpha, \beta} M$ be the colimit of the diagram of topological spaces consisting of the topological spaces $\left[\alpha_{0} \ldots \alpha_{p}\right]$ and $\left[\alpha_{0} \ldots \alpha_{p}\right]_{i}$ with $\alpha_{0}=\alpha$ and $\alpha_{p}=\beta$ with the two kinds of maps above defined. The composition law of $A$ and the free concatenation obviously defines a continuous associative map $\mathbb{P}_{\alpha, \beta} M \times \mathbb{P}_{\beta, \gamma} M \longrightarrow \mathbb{P}_{\alpha, \gamma} M$.

Proposition 15.1. One has the pushout diagram of flows

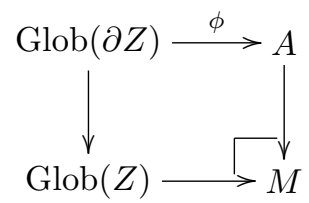

Proof. Let us consider a commutative diagram like:

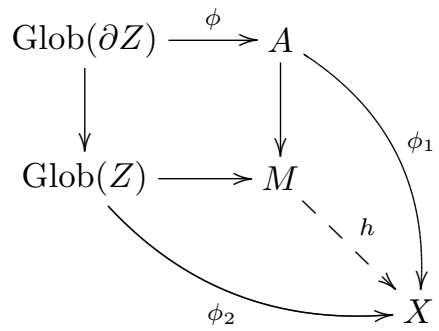

One has to prove that there exists $h$ making everything commutative. We do not have any choice for the definition on the 0-skeleton: $h(\alpha)=\phi_{1}(\alpha)$. The diagram of flows above gives a commutative diagram of topological spaces

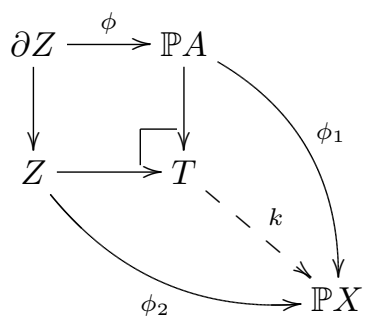

By construction of $T$, there exists a continuous map $k: T \longrightarrow \mathbb{P}_{h(\phi(0)), h(\phi(1))} X \subset$ $\mathbb{P} X$ making the diagram commutative.

Constructing a continuous map $\mathbb{P} M \longrightarrow \mathbb{P} X$ is equivalent to constructing continuous maps $\left[\alpha_{0} \ldots \alpha_{p}\right] \longrightarrow \mathbb{P}_{h\left(\alpha_{0}\right), h\left(\alpha_{p}\right)} X$ and $\left[\alpha_{0} \ldots \alpha_{p}\right]_{i} \longrightarrow \mathbb{P}_{h\left(\alpha_{0}\right), h\left(\alpha_{p}\right)} X$ for any finite sequence $\alpha_{0} \ldots \alpha_{p}$ of $\mathcal{M}$ such that any diagram like

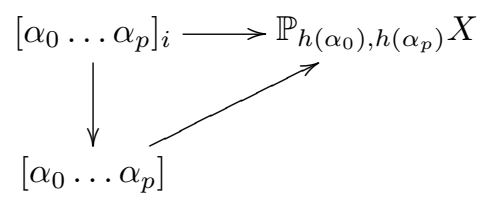


or like

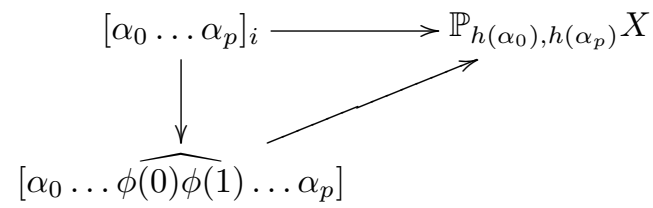

is commutative. There are such obvious maps by considering the continuous maps $Z_{\alpha, \beta} \longrightarrow \mathbb{P}_{h(\alpha), h(\beta)} X$ and by composing with the composition law of $X$. Hence the result.

Theorem 15.2. Suppose that one has the pushout of flows

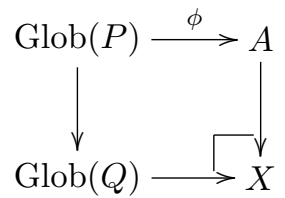

where $P \longrightarrow Q$ is an inclusion of a deformation retract of topological spaces. Then the continuous map $\mathbb{P} f: \mathbb{P} A \longrightarrow \mathbb{P} X$ is a weak homotopy equivalence.

Proof. Let us start with the diagram $\mathcal{D}=\mathcal{D}_{0}$ of topological spaces constructed for Proposition 15.1 calculating $\mathbb{P} X$. We are going to modify $\mathcal{D}$, by transfinite induction, in order to obtain another diagram of topological spaces, whose colimit will still be isomorphic to $\mathbb{P} X$ and such that all arrows will be inclusions of a deformation retract.

We are going to add vertices and arrows to the diagram above in the following way. For any configuration like

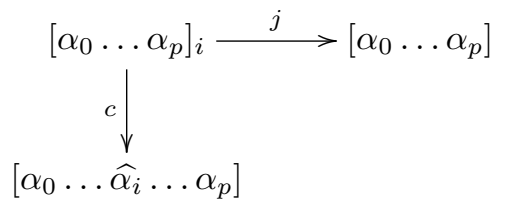

where $c$ is induced by the composition law of $A$ and $j$ is the unique possible inclusion of a deformation retract, let us draw the cocartesian square

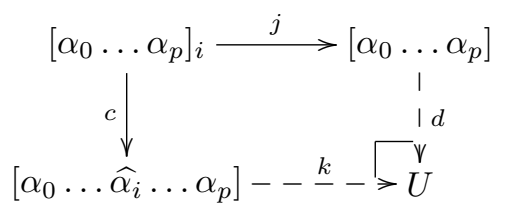

Notice that $k$ is an inclusion of a deformation retract because the class of inclusions of a deformation retract is closed under pushout : cf. [27] for an elementary proof, or [35] for a model-categoric argument. Indeed, an inclusion of a deformation retract is a trivial cofibration for the Strøm model category of compactly generated topological spaces. So the corresponding class is closed under pushout because it coincides with the class of morphisms satisfying the LLP with respect to any Hurewicz fibration. 
One will say that the maps $j$ and $k$ are orthogonal to the composition law of $A$ and that the maps $c$ and $d$ are parallel to the composition law of $A$. Repeat the process for any configuration like

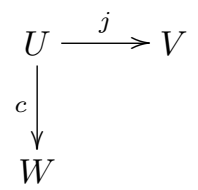

where $j$ is orthogonal to the composition law of $A$ and $c$ parallel to the composition law of $A$ by completing the configuration by a cocartesian square of topological spaces

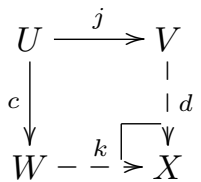

By induction, one will say that $k$ is orthogonal to the composition law and that $d$ is parallel to the composition law. Notice that, in this diagram, any map which is orthogonal to the composition law is an inclusion of a deformation retract of topological spaces. At each step consisting of adding an object so that it creates a pushout square in the diagram, one obtains a diagram $\mathcal{D}_{\lambda+1}$ from a diagram $\mathcal{D}_{\lambda}$. There is a canonical continuous map $\lim _{\longrightarrow} \mathcal{D}_{\lambda} \longrightarrow \lim \mathcal{D}_{\lambda+1}$ which is an homeomorphism.

Let us say that the topological spaces $\left[\alpha_{0} \ldots \alpha_{p}\right]$ and $\left[\alpha_{0} \ldots \alpha_{p}\right]_{i}$ are of length $p$. By induction, one defines the length of a topological space as being constant along the arrows orthogonal to the composition law of $A$. The length is strictly decreasing along the arrows parallel to the composition law of $A$. Therefore the process stops after an, eventually, transfinite number of steps. Moreover the only map which can starts from an element of length 1 is an arrow orthogonal to the composition law of $A$. Therefore such a map is necessarily an inclusion of a deformation retract of topological spaces.

Let us say that the process stops for $\lambda=\lambda_{0}$. For any vertex $v$ of $\mathcal{D}_{\lambda_{0}}$, there exists an arrow $v \longrightarrow w$ of $\mathcal{D}_{\lambda_{0}}$ with $w$ of length 1 . Therefore the colimit of the diagram $\mathcal{D}_{\lambda_{0}}$ is isomorphic to the colimit of the subdiagram of $\mathcal{D}_{\lambda_{0}}$ consisting of the vertex of length 1.

The initial diagram $\mathcal{D}=\mathcal{D}_{0}$ has therefore the same colimit as a diagram of topological spaces of the form a concatenation of straight lines of the form

$$
\mathbb{P}_{\alpha, \beta} A \longrightarrow M_{1} \longrightarrow M_{2} \longrightarrow \ldots
$$

where all arrows are inclusions of a deformation retract. Therefore $\mathbb{P} A \longrightarrow \mathbb{P} X$ is a weak homotopy equivalence since any inclusion of a deformation retract is a closed $T_{1}$ inclusion and a weak homotopy equivalence and since any transfinite composition of such maps is a weak homotopy equivalence (cf [27] Lemma 2.4.5, Corollary 2.4.6 and Lemma 2.4.8). 
16. $J^{g l}-\operatorname{cell} \subset I_{+}^{g l}-\operatorname{cof} \cap \mathcal{S}$

Proposition 16.1. One has $J^{g l}-\operatorname{cell} \subset I^{g l}-\operatorname{cof} \cap \mathcal{S}$.

Proof. The continuous maps $\mathbf{D}^{n} \cong \mathbf{D}^{n} \times\{0\} \longrightarrow \mathbf{D}^{n} \times[0,1]$ are inclusions of a deformation retract for any $n \geqslant 0$. So by Theorem $15.2, J^{g l}-$ cell $\subset \mathcal{S}$. The class $I^{g l}-c o f$ is closed under pushout and transfinite composition. So it then suffices to prove that $J^{g l} \subset I^{g l}-c o f$. A morphism of flows $f: X \longrightarrow Y$ satisfies the RLP with respect to $\operatorname{Glob}\left(\mathbf{D}^{n}\right) \longrightarrow \operatorname{Glob}\left(\mathbf{D}^{n} \times[0,1]\right)$ if and only if for any $\alpha, \beta \in X^{0}, \mathbb{P}_{\alpha, \beta} X \longrightarrow \mathbb{P}_{f(\alpha), f(\beta)} Y$ is a Serre fibration by Proposition 13.2. But again by Proposition 13.2, for any element $f: X \longrightarrow Y$ of $I^{g l}-i n j$, for any $\alpha, \beta \in X^{0}, \mathbb{P}_{\alpha, \beta} X \longrightarrow \mathbb{P}_{f(\alpha), f(\beta)} Y$ is a trivial Serre fibration, so a Serre fibration. Hence the result.

Proposition 16.2. Let $f$ be a morphism of flows. Then the following conditions are equivalent:

1. $f$ is synchronized

2. $f$ satisfies the $R L P$ with respect to $R:\{0,1\} \longrightarrow\{0\}$ and $C: \varnothing \subset\{0\}$.

Proof. Let $f: X \longrightarrow Y$ that satisfies the RLP with respect to $R:\{0,1\} \longrightarrow\{0\}$ and $C:\{0\} \subset\{0,1\}$. Let us suppose that $f(a)=f(b)$ for some $a, b \in X^{0}$. Then consider the commutative diagram

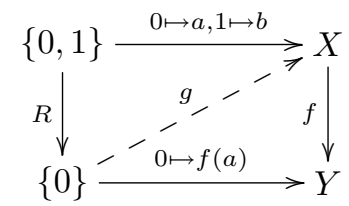

By hypothesis, there exists $g$ making both triangles commutative. So $b=g \circ R(1)=$ $g(0)=g \circ R(0)=a$. So $f$ induces a one-to-one map on the 0 -skeletons. Now take $a \in Y^{0}$. Then consider the commutative diagram

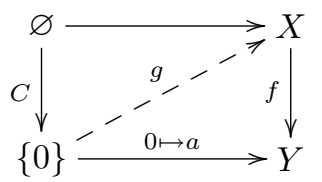

By hypothesis, there exists $g$ making both triangles commutative. Then $a=f(g(1))$. So $f$ induces an onto map on the 0 -skeletons. Therefore condition 2 implies condition 1. Conversely, if $f$ is synchronized, let $\left(f^{0}\right)^{-1}: Y^{0} \longrightarrow X^{0}$ be the inverse of the restriction $f^{0}$ of $f$ to the 0 -skeleton. Consider a commutative diagram like

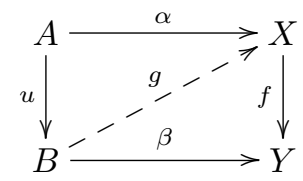

where $A$ and $B$ are two flows such that $A=A^{0}, B=B^{0}$ and where $u$ is any set map. 
Then $g=\left(f^{0}\right)^{-1} \circ \beta$ makes both triangles commutative. Indeed $f \circ\left(f^{0}\right)^{-1} \circ \beta=\beta$ and $\left(f^{0}\right)^{-1} \circ \beta \circ u=\left(f^{0}\right)^{-1} \circ f \circ \alpha=\alpha$.

Corollary 16.3. $J^{g l}-$ cell $\subset I_{+}^{g l}-\operatorname{cof} \cap \mathcal{S}$.

Proof. The elements of $I^{g l}-$ cof satisfies the LLP with respect to any element of $I^{g l}-i n j$. So in particular, the elements of $I^{g l}-$ cof satisfies the LLP with respect to any synchronized element of $I^{g l}-i n j$. But a synchronized element of $I^{g l}-i n j$ is precisely an element of $I_{+}^{g l}-i n j$ by Proposition 16.2. Therefore $I^{g l}-$ cof $\subset$ $I_{+}^{g l}-\operatorname{cof}$.

17. $I_{+}^{g l}-i n j=J^{g l}-i n j \cap \mathcal{S}$

Proposition 17.1. Any morphism of $I_{+}^{g l}-i n j$ is a trivial fibration. In other terms, $I_{+}^{g l}-i n j \subset J^{g l}-i n j \cap \mathcal{S}$.

Proof. Let $f: X \longrightarrow Y$ be a morphism of flows with $f \in I_{+}^{g l}-i n j$. Then $f$ is synchronized by Proposition 16.2. By Proposition 13.2, for any $\alpha, \beta \in X^{0}$, $\mathbb{P}_{\alpha, \beta} X \longrightarrow \mathbb{P}_{f(\alpha), f(\beta)} Y$ is a trivial Serre fibration. So $f$ is a weak S-homotopy equivalence. And again by Proposition 13.2, this implies that $f$ satisfies the RLP with respect to $J^{g l}$. Hence the result.

Proposition 17.2. Any trivial fibration is in $I_{+}^{g l}-i n j$. In other terms, $J^{g l}-i n j \cap$ $\mathcal{S} \subset I_{+}^{g l}-i n j$.

Proof. Let $f$ be a trivial fibration. By Proposition 13.2, for any $\alpha, \beta \in X^{0}$, the continuous map $\mathbb{P}_{\alpha, \beta} X \longrightarrow \mathbb{P}_{f(\alpha), f(\beta)} Y$ is a fibration. But $f \in \mathcal{S}$. Therefore the fibrations $\mathbb{P}_{\alpha, \beta} X \longrightarrow \mathbb{P}_{f(\alpha), f(\beta)} Y$ are trivial. So by Proposition $13.2, f$ satisfies the RLP with respect to $I^{g l}$. Since $f$ is also synchronized, then $f$ satisfies the RLP with respect to $R$ and $C$ as well.

\section{The model structure of Flow}

Corollary 18.1. The category of flows together with the weak S-homotopy equivalences, the cofibrations and the fibrations is a model category. The cofibrations are the retracts of the elements of $I_{+}^{g l}-$ cell. Moreover, any flow is fibrant.

Proof. The first part of the statement is a consequence of Proposition 11.5, Proposition 16.1, Proposition 17.1, Proposition 17.2 and Theorem 12.5. It remains to prove that any flow is fibrant. Let $X$ be a flow. Let $\mathbf{1}$ be the flow such that $\mathbf{1}^{0}=\{0\}$ and $\mathbb{P} \mathbf{1}=\{1\}$. Then $\mathbf{1}$ is a terminal object of Flow. Consider a commutative diagram like

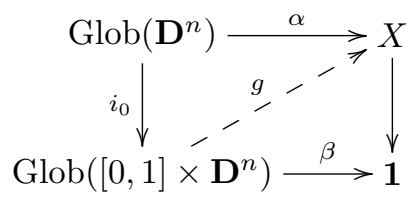


Let $g(0)=\alpha(0), g(1)=\alpha(1)$ and $g(t, z)=\alpha(z)$ for any $(t, z) \in[0,1] \times \mathbf{D}^{n}$. Then $g$ makes both triangles commutative.

Corollary 18.2. Any cofibration for this model structure induces a closed inclusion between path spaces.

Proof. This is a consequence of Corollary 9.8 and of Corollary 18.1.

\section{S-homotopy and the model structure of Flow}

In any model category, the canonical morphism $X \sqcup X \longrightarrow X$ factors as a cofibration $X \sqcup X \longrightarrow I(X)$ and a trivial fibration $I(X) \longrightarrow X$. One then says that two morphisms $f$ and $g$ from $X$ to $Y$ are left homotopy equivalent (this situation being denoted by $f \sim_{l} g$ ) if and only if there exists a morphism $I(X) \longrightarrow Y$ such that the composite $X \sqcup X \longrightarrow I(X) \longrightarrow Y$ is exactly $f \sqcup g$. On cofibrant and fibrant objects, the left homotopy is an equivalence relation simply called homotopy. Then one can say that two cofibrant and fibrant flows $X$ and $Y$ are left homotopy equivalent (this situation being denoted by $X \sim_{l} Y$ ) if and only if there exists a morphism of flows $f: X \longrightarrow Y$ and a morphism of flows $g: Y \longrightarrow X$ such that $f \circ g \sim_{l} \operatorname{Id}_{Y}$ and $g \circ f \sim_{l} \operatorname{Id}_{X}$.

Theorem 19.1. Two cofibrant flows are left homotopy equivalent if and only if they are S-homotopy equivalent.

The similar fact is trivial in Top because for any cofibrant topological space $X$, the continuous map $X \sqcup X \longrightarrow[0,1] \times X$ sending one copy of $X$ to $\{0\} \times X$ and the other one to $\{1\} \times X$ is a relative $I$-cell complex, and therefore a cofibration for the model structure of Top, and the continuous projection map $[0,1] \times X \longrightarrow X$ is a fibration. A similar situation does not hold in the framework of flows.

Proposition 19.2. There exists a cofibrant flow $X$ such that the canonical morphism of flows $[0,1] \otimes X \longrightarrow X$ such that $t \otimes x \mapsto x$ is not a fibration for the model structure of Flow.

Proof. Let $X^{0}$ be the three-element set $\{\alpha, \beta, \gamma\}$. Let $\mathbb{P}_{\alpha, \beta} X=\{u\}, \mathbb{P}_{\beta, \gamma} X=\{v\}$, and $\mathbb{P}_{\alpha, \gamma} X=\mathbf{D}^{1}$ with the relation $1=u * v$. Consider the commutative diagram

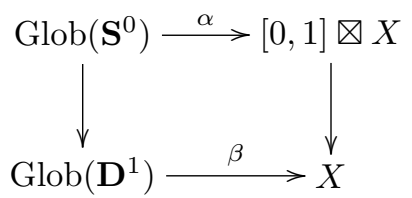

with $\alpha(-1)=0 \bigotimes-1, \alpha(1)=(0 \otimes u) *(1 \otimes v)$ and $\beta(z)=z$ for $z \in \mathbf{D}^{1}$. Suppose that there exists $g: \operatorname{Glob}\left(\mathbf{D}^{1}\right) \longrightarrow[0,1] \otimes X$ making the above diagram commutative. For $z \in \mathbf{D}^{1} \backslash\{1\}$, then the execution path $t \otimes z$ of $[0,1] \otimes X$ is composable with nothing by construction. So for such $z, g(z)=\phi(z) \otimes z$ for some continuous map $\phi: \mathbf{D}^{1} \backslash\{1\} \longrightarrow[0,1]$. Then $n \mapsto \phi(1-1 /(n+1))$ is a sequence of $[0,1]$ and so contains a subsequence converging to some $t_{0} \in[0,1]$. Then $(0 \otimes u) *(1 \otimes v)=t_{0} \otimes 1$, which contredicts the explicit description of $[0,1] \otimes X$ of Corollary 8.3. 
Definition 19.3. Let $X$ be a flow. Then the flow $\square X$ is defined by the cocartesian diagram

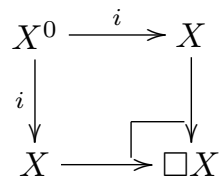

where $i: X^{0} \longrightarrow X$ is the canonical inclusion. This flow is called the square of $X$.

Proposition 19.4. For any flow $X$, the canonical morphism of flows $k_{X}: X \sqcup$ $X \longrightarrow \square X$ is a cofibration.

Proof. This map is indeed an (eventually transfinite) composition of pushouts of $R:\{0,1\} \longrightarrow\{0\}$, so an element of $I_{+}^{g l}-$ cell $\subset I_{+}^{g l}-$ cof.

Proposition 19.5. Let $X$ be a cofibrant flow. Then the canonical morphism of flows $j_{X}: X \sqcup X \longrightarrow[0,1] \otimes X$ induced by the inclusions $X \cong\{0\} \otimes X \subset[0,1] \otimes X$ and $X \cong\{1\} \otimes X \subset[0,1] \otimes X$ is a cofibration.

Proof. The morphism $j_{X}$ factors as $j_{X}=\ell_{X} \circ k_{X}$. Using Proposition 19.4, it suffices to prove that $\ell_{X}: \square X \longrightarrow[0,1] \otimes X$ is a cofibration. Both functors $X \mapsto \square X$ and $X \mapsto[0,1] \otimes X$ commute with colimits and a colimit of cofibrations is a cofibration. So it suffices to prove that for any CW-complex $Z, \ell_{\mathrm{Glob}(Z)}: \square \operatorname{Glob}(Z) \longrightarrow[0,1] \otimes$ $\operatorname{Glob}(Z)$ is a cofibration. $\operatorname{But} \square \operatorname{Glob}(Z) \cong \operatorname{Glob}(Z \sqcup Z)$ and $[0,1] \otimes \operatorname{Glob}(Z) \cong$ $\operatorname{Glob}([0,1] \times Z)$. Since $Z \sqcup Z \longrightarrow[0,1] \times Z$ is a cofibration in Top, then it is a retract of an element of $I-$ cell. So $\ell_{\mathrm{Glob}(Z)}$ is a retract of an element of $I^{g l}-$ cell. Therefore $\ell_{\mathrm{Glob}(Z)}$ is a cofibration of flows.

Proposition 19.6. Let $f: X \longrightarrow Y$ be a morphism of flows. If $f$ is a S-homotopy equivalence, then it is synchronized and for any $\alpha, \beta \in X^{0}$, the continuous map $\mathbb{P}_{\alpha, \beta} f: \mathbb{P}_{\alpha, \beta} X \longrightarrow \mathbb{P}_{f(\alpha), f(\beta)} Y$ is an homotopy equivalence.

Proof. Let $g: Y \longrightarrow X$ be a S-homotopic inverse of $f$. Let $F:[0,1] \otimes X \longrightarrow X$ be a S-homotopy from $g \circ f$ to $\operatorname{Id}_{X}$. Let $G:[0,1] \otimes Y \longrightarrow Y$ be a S-homotopy from $f \circ g$ to $\operatorname{Id}_{Y}$. Let $\alpha, \beta \in X^{0}$. Then the composite

$$
F^{\prime}: \operatorname{Glob}\left([0,1] \times \mathbb{P}_{\alpha, \beta} X\right) \cong[0,1] \otimes \operatorname{Glob}\left(\mathbb{P}_{\alpha, \beta} X\right) \longrightarrow[0,1] \otimes X \longrightarrow X
$$

defined by

$$
\begin{aligned}
& F^{\prime}(0)=f(\alpha) \text { and } F^{\prime}(1)=f(\beta) \\
& F^{\prime}(t, x)=F(t \otimes x) \text { for }(t, x) \in[0,1] \times \mathbb{P}_{\alpha, \beta} X
\end{aligned}
$$

yields an homotopy from $\mathbb{P}_{\alpha, \beta} g \circ \mathbb{P}_{\alpha, \beta} f$ to $\operatorname{Id}_{\mathbb{P}_{\alpha, \beta} X}$. In the same way, one constructs from $G$ an homotopy from $\mathbb{P}_{\alpha, \beta} f \circ \mathbb{P}_{\alpha, \beta} g$ to $\operatorname{Id}_{\mathbb{P}_{\alpha, \beta} Y}$.

Proof of Theorem 19.1. The canonical morphism of flows $[0,1] \otimes X \longrightarrow X$ factors as a cofibration $i_{X}:[0,1] \otimes X \longrightarrow I(X)$ followed by a trivial fibration $p_{X}: I(X) \longrightarrow X$. So the canonical morphism $X \sqcup X \longrightarrow X$ factors as a cofibration $i_{X} \circ j_{X}: X \sqcup X \longrightarrow$ $I(X)$ followed by a trivial fibration $p_{X}: I(X) \longrightarrow X$. 
Now let $X$ and $Y$ be two S-homotopy equivalent cofibrant flows. Let $f: X \longrightarrow Y$ be a S-homotopy equivalence between $X$ and $Y$. Then $f$ is a weak S-homotopy equivalence by Proposition 19.6. So $X$ and $Y$ are left-homotopy equivalent. Reciprocally, let $X$ and $Y$ be two left-homotopy equivalent cofibrant flows. Therefore there exists a morphism of flows $f: X \longrightarrow Y$ and a morphism of flows $g: Y \longrightarrow X$ such that $f \circ g \sim_{l} \operatorname{Id}_{Y}$ and $g \circ f \sim_{l} \operatorname{Id}_{X}$. Then there exists morphisms of flows $H_{X}: I(X) \longrightarrow X$ and $H_{Y}: I(Y) \longrightarrow Y$ such that $H_{X} \circ i_{X} \circ j_{X}=(g \circ f) \sqcup \operatorname{Id}_{X}$ and $H_{Y} \circ i_{Y} \circ j_{Y}=(f \circ g) \sqcup \operatorname{Id}_{Y}$. So $H_{X} \circ i_{X}$ is a S-homotopy between $g \circ f$ and $\operatorname{Id}_{X}$ and $H_{Y} \circ i_{Y}$ is a S-homotopy between $f \circ g$ and $\operatorname{Id}_{Y}$. Therefore $X$ and $Y$ are S-homotopy equivalent.

One obtains finally the following theorem:

Theorem 19.7. There exists a structure of model category on Flow such that

1. The weak equivalences are the weak S-homotopy equivalences.

2. The fibrations are the morphisms of flows that satisfy the RLP with respect to the morphisms of flows $\operatorname{Glob}\left(\mathbf{D}^{n}\right) \longrightarrow \operatorname{Glob}\left([0,1] \times \mathbf{D}^{n}\right)$ induced by the maps $x \mapsto(0, x)$.

3. The cofibrations are the morphisms of flows that satisfy the LLP with respect to any morphism of flows that satisfies the RLP with respect to the morphisms of flows $\operatorname{Glob}\left(\mathbf{S}^{n-1}\right) \longrightarrow \operatorname{Glob}\left(\mathbf{D}^{n}\right)$ induced by the inclusions $\mathbf{S}^{n-1} \subset \mathbf{D}^{n}$ and with respect to $R:\{0,1\} \longrightarrow\{0\}$ and $C: \varnothing \subset\{0\}$.

4. Any flow is fibrant.

5. The fibration are the morphism of flows inducing a Serre fibration of topological spaces between path spaces.

6. Two cofibrant flows are homotopy equivalent for this model structure if and only if they are S-homotopy equivalent.

Corollary 19.8. Let $X$ and $Y$ be two cofibrant flows. Let $f: X \longrightarrow Y$ be a synchronized morphism of flows. Then the following conditions are equivalent:

1. for any $\alpha, \beta \in X^{0}$, the continuous map $\mathbb{P}_{\alpha, \beta} X \longrightarrow \mathbb{P}_{f(\alpha), f(\beta)} Y$ is a weak homotopy equivalence

2. for any $\alpha, \beta \in X^{0}$, the continuous map $\mathbb{P}_{\alpha, \beta} X \longrightarrow \mathbb{P}_{f(\alpha), f(\beta)} Y$ is homotopy equivalence

3. $f$ is a weak S-homotopy equivalence

4. $f$ is a S-homotopy equivalence.

Question 19.9. How to find two flows $X$ and $Y$ (necessarily not cofibrant) and a synchronized morphism of flows $f: X \longrightarrow Y$ which is not a S-homotopy equivalence and such that for any $\alpha, \beta \in X^{0}, f$ induces an homotopy equivalence from $\mathbb{P}_{\alpha, \beta} X$ to $\mathbb{P}_{f(\alpha), f(\beta)} Y$.

\section{Why no identity maps in the notion of flow ?}

There exist several reasons. Here is one of them. The section "Why non-contracting maps ?" of [20] is also related to this question. A similar phenomenon appears 
in the construction of the "corner homology" of an $\omega$-category in $[\mathbf{1 3}]$ (cf. Proposition 4.2 of the latter paper).

Let $X$ be a flow. Let us consider the topological space $\mathbb{P}^{-} X$ which is solution of the following universal problem: there exists a continuous map $h^{-}: \mathbb{P} X \longrightarrow \mathbb{P}^{-} X$ such that $h(x * y)=h(x)$ and any continuous map $f: \mathbb{P} X \longrightarrow Y$ such that $f(x * y)=f(x)$ factors uniquely as a composite $\bar{f} \circ h^{-}$for a unique continuous map $\bar{f}: \mathbb{P}^{-} X \longrightarrow Y$. And let us consider the topological space $\mathbb{P}^{+} X$ which is solution of the following universal problem: there exists a continuous map $h^{+}: \mathbb{P} X \longrightarrow \mathbb{P}^{+} X$ such that $h(x * y)=h(y)$ and any continuous map $f: \mathbb{P} X \longrightarrow Y$ such that $f(x * y)=f(y)$ factors uniquely as a composite $\bar{f} \circ h^{+}$for a unique continuous map $\bar{f}: \mathbb{P}^{+} X \longrightarrow Y$. The space $\mathbb{P}^{-} X$ is called the branching space of $X$ and the space $\mathbb{P}^{+} X$ is called the merging space of $X$. Both mappings $\mathbb{P}^{-}:$Flow $\longrightarrow$ Top and $\mathbb{P}^{+}:$Flow $\longrightarrow$ Top are crucial for the definition of T-homotopy (cf. [17] [11]).

Suppose now that a flow $X$ is a small category enriched over the category of compactly generated topological spaces Top, that is we suppose that there exists an additional continuous map $i: X^{0} \longrightarrow \mathbb{P} X$ with $s(i(\alpha))=\alpha$ and $t(i(\alpha))=\alpha$ for any $\alpha \in X^{0}$. Then for any $x \in \mathbb{P} X$, we would have $x=s(x) * x$ and $x=x * t(x)$. So both topological spaces $\mathbb{P}^{-} X$ and $\mathbb{P}^{+} X$ would be discrete. Therefore, in such a setting, the correct definition would be for $\mathbb{P}^{-} X$ (resp. $\left.\mathbb{P}^{+} X\right)$ the quotient of $\mathbb{P} X \backslash i\left(X^{0}\right)$ by the identifications $x=x * y$ (resp. $y=x * y$ ). But with such a definition, the mappings $X \mapsto \mathbb{P}^{-} X$ and $X \mapsto \mathbb{P}^{+} X$ cannot be functorial anymore.

\section{Concluding discussion}

If $Z$ is a cofibrant topological space, then $\operatorname{Glob}(Z)$ is a cofibrant flow. Let us denote by $\mathbf{T o p}_{c}$ the full and faithful subcategory of cofibrant topological spaces. Let us denote by Flow ${ }_{c}$ the full and faithful subcategory of cofibrant flows. Then one has the commutative diagram of functors

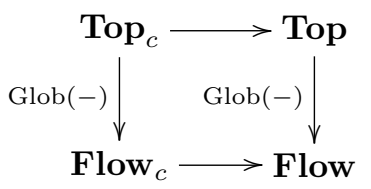

which becomes the commutative diagram of functors

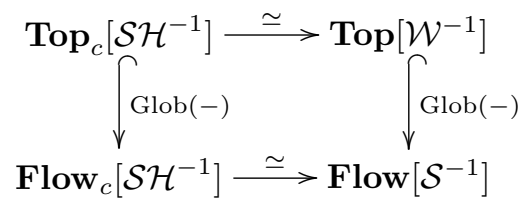

where $\mathcal{S H}$ is the class of homotopy equivalences (of topological spaces or of flows), $\mathcal{W}$ the class of weak homotopy equivalences of topological spaces, and at last $\mathcal{S}$ the class of weak S-homotopy equivalences of flows. Both horizontal arrows of the latter diagram are equivalence of categories. The notation $\mathcal{C}\left[\mathcal{X}^{-1}\right]$ means of course the localization of the category $\mathcal{C}$ with respect to the class of morphisms $\mathcal{X}$. 


\section{References}

[1] Al-Agl, F. A., R. Brown and R. Steiner, Multiple categories: the equivalence of a globular and a cubical approach, Adv. Math. 170 (2002), pp. 71-118.

[2] Borceux, F., "Handbook of categorical algebra. 1," Cambridge University Press, Cambridge, 1994, xvi+345 pp., basic category theory.

[3] Brown, R., "Topology," Ellis Horwood Ltd., Chichester, 1988, second edition, xviii +460 pp., a geometric account of general topology, homotopy types and the fundamental groupoid.

[4] Brown, R. and P. J. Higgins, Colimit theorems for relative homotopy groups, Journal of Pure and Applied Algebra (1981), pp. 11-41.

[5] Brown, R. and P. J. Higgins, On the algebra of cubes, J. Pure Appl. Algebra 21 (1981), pp. 233-260.

[6] Coffman, E. G., M. J. Elphick and A. Shoshani, System deadlocks, Computing Surveys 3 (1971), pp. 67-78.

[7] Cridlig, R., Implementing a static analyzer of concurrent programs: Problems and perspectives, in: Logical and Operational Methods in the Analysis of Programs and Systems, 1996, pp. 244-259.

[8] Dijkstra, E., "Cooperating Sequential Processes," Academic Press, 1968.

[9] Dwyer, B., P. S. Hirschhorn and D. Kan, Model categories and more general abstract homotopy theory (1997), available at http://wwwmath.mit.edu/ ${ }^{\sim} \mathrm{psh} /$.

[10] Fajstrup, L., E. Goubault and M. Raußen, Detecting deadlocks in concurrent systemsin: CONCUR'98: concurrency theory (Nice), Lecture Notes in Comput. Sci. 1466, Springer, Berlin, 1998 pp. 332-347.

[11] Gaucher, P., Homotopy branching space and weak dihomotopy, arXiv:math.AT/0304112.

[12] Gaucher, P., From concurrency to algebraic topology, in: Electronic Notes in Theoretical Computer Science, 392 (2000), p. 19.

[13] Gaucher, P., Homotopy invariants of higher dimensional categories and concurrency in computer science, Math. Structures Comput. Sci. 10 (2000), pp. $481-524$.

[14] Gaucher, P., Combinatorics of branchings in higher dimensional automata, Theory and Applications of Categories 8 (2001), pp. 324-376.

[15] Gaucher, P., About the globular homology of higher dimensional automata, Cahiers Topologie Géom. Différentielle Catég. 43 (2002), pp. 107-156.

[16] Gaucher, P., The branching nerve of HDA and the Kan condition, Theory and Applications of Categories 11 (2003), pp. 75-106. 
[17] Gaucher, P., Comparing globular CW-complex and flow (2003), arxiv:math.AT/0308063.

[18] Gaucher, P., Concurrent process up to homotopy (I), C. R. Acad. Sci. Paris Ser. I Math. 336 (2003), pp. 593-596, French.

[19] Gaucher, P., Concurrent process up to homotopy (II), C. R. Acad. Sci. Paris Ser. I Math. 336 (2003), pp. 647-650, French.

[20] Gaucher, P. and E. Goubault, Topological deformation of higher dimensional automata, Homology, Homotopy and Applications 5 (2003), pp. p.39-82.

[21] Goerss, P. G. and J. F. Jardine, "Simplicial homotopy theory," Birkhäuser Verlag, Basel, 1999, xvi+510 pp.

[22] Goubault, E., "The Geometry of Concurrency," Ph.D. thesis, École Normale Supérieure (1995).

[23] Goubault, E., Some geometric perspectives in concurrency theory, Homology, Homotopy and Applications 5 (2003), pp. p.95-136.

[24] Guitart, R. and L. Van den Bril, Décompositions et lax-complétions, Cahiers Topologie Géom. Differentielle 18 (1977), pp. 333-407.

[25] Gunawardena, J., Homotopy and concurrency, Bull. EATCS 54 (1994), pp. 184-193.

[26] Hirschhorn, P. S., "Model categories and their localizations," Mathematical Surveys and Monographs 99, American Mathematical Society, Providence, RI, 2003, xvi+457 pp.

[27] Hovey, M., "Model categories," American Mathematical Society, Providence, RI, 1999, xii+209 pp.

[28] Lewis, L. G., "The stable category and generalized Thom spectra," Ph.D. thesis, University of Chicago (1978).

[29] Mac Lane, S., "Categories for the working mathematician," Springer-Verlag, New York, 1998, second edition, xii+314 pp.

[30] May, J. P., "A concise course in algebraic topology," University of Chicago Press, Chicago, IL, 1999, x+243 pp.

[31] McCord, M. C., Classifying spaces and infinite symmetric products, Trans. Amer. Math. Soc. 146 (1969), pp. 273-298.

[32] Pratt, V., Modeling concurrency with geometry, in: Proc. 18th Ann. ACM Symposium on Principles of Programming Languages, 1991, pp. 311-322.

[33] Steenrod, N. E., A convenient category of topological spaces, Michigan Math. J. 14 (1967), pp. 133-152.

[34] Street, R., The algebra of oriented simplexes, J. Pure Appl. Algebra 49 (1987), pp. 283-335. 
[35] Strøm, A., The homotopy category is a homotopy category, Arch. Math. (Basel) 23 (1972), pp. 435-441.

[36] Vogt, R. M., Convenient categories of topological spaces for algebraic topology, in: Proceedings of the Advanced Study Institute on Algebraic Topology (1970), Vol. III (1970), pp. 649-656. Various Publ. Ser., No. 13.

[37] Vogt, R. M., Convenient categories of topological spaces for homotopy theory, Arch. Math. (Basel) 22 (1971), pp. 545-555.

This article may be accessed via WWW at http://www.rmi.acnet.ge/hha/ or by anonymous ftp at

ftp://ftp.rmi.acnet.ge/pub/hha/volumes/2003/n1a20/v5n1a20.(dvi,ps,pdf)

Philippe Gaucher gaucher@pps.jussieu.fr

http://www.pps.jussieu.fr/ ̃aucher/

Preuves Programmes et Systèmes

Université Paris 7-Denis Diderot

Case 7014

2 Place Jussieu

75251 PARIS Cedex 05

France 